Linköping Studies in Science and Technology. Licenciate Thesis.

No. 1709

\title{
A Classical-Light Attack on Energy-Time Entangled Quantum Key Distribution, and Countermeasures
}

Jonathan Jogenfors
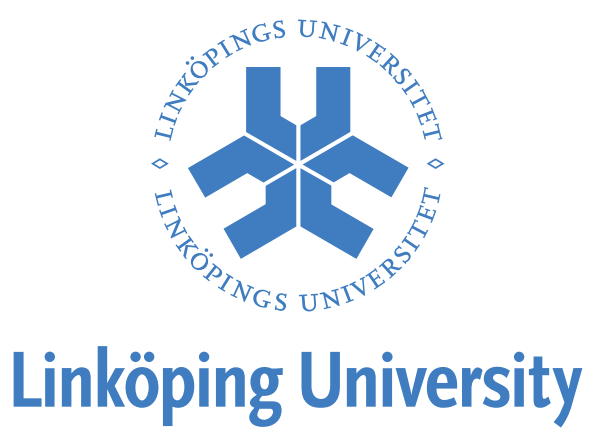

INSTITUTE OF TECHNOLOGY

Institutionen för Systemteknik

Linköpings Universitet, 58183 Linköping, Sweden

Linköping 2015 



\title{
A Classical-Light Attack on Energy-Time Entangled Quantum Key Distribution, and Countermeasures
}

\author{
Jonathan Jogenfors
}

Linköping Studies in Science and Technology. Licenciate Thesis. No. 1709

Copyright (c) Jonathan Jogenfors 2015 unless otherwise noted.

$$
\text { ISBN 978-91-7519-118-8 }
$$

ISSN 0280-7971

Printed by LiU-Tryck in Linköping, Sweden 2015

This is a Swedish Licentiate Thesis. The Licentiate degree comprises 120 ECTS credits of postgraduate studies. 
To Anna 


\section{Abstract}

Quantum key distribution (QKD) is an application of quantum mechanics that allows two parties to communicate with perfect secrecy. Traditional QKD uses polarization of individual photons, but the development of energytime entanglement could lead to QKD protocols robust against environmental effects. The security proofs of energy-time entangled QKD rely on a violation of the Bell inequality to certify the system as secure. This thesis shows that the Bell violation can be faked in energy-time entangled QKD protocols that involve a postselection step, such as Franson-based setups. Using pulsed and phase-modulated classical light, it is possible to circumvent the Bell test which allows for a local hidden-variable model to give the same predictions as the quantum-mechanical description. We show that this attack works experimentally and also how energy-time-entangled systems can be strengthened to avoid our attack. 


\section{Populärvetenskaplig sammanfattning}

Kvantkryptering är en tillämpning av kvantmekanik där fysikens lagar används för att kryptera information. Till skillnad mot klassisk kryptering, som bygger på matematiska problem som antas (men inte bevisats) vara svåra att forcera, kan kvantkryptering ge garanterad säkerhet. Inte ens en oändligt snabb dator kan kringgå hemligheter som krypterats på kvantmekanisk väg eftersom säkerheten kommer direkt från fysikens lagar. Vanligtvis bygger kvantkryptering på enstaka fotoner där polarisering bär information, men nackdelen med den metoden är att polarisering är relativt känslig för störningar. Därför har det på senare tid kommit en ny metod, energi-tidssnärjning, som antas vara mer robust och därmed kan vara mer lämpat till att användas i stor skala. Det mest kända protokollet som bygger på denna teknik är Fransons interferometer. Detta system har utvärderats av ledande forskargrupper runt om i världen, och under många år har man trott att det kan uppnå garanterad säkerhet. Denna avhandling kommer dock visa på en inbyggd, allvarlig svaghet som gör att en tredje part kan forcera säkerheten i Fransons interferometer utan att lämna spår efter sig. Följderna är påtagliga; kvantkryptering som baseras på Fransons interferometer kan avlyssnas och måste därför byta ut den grundläggande säkerhetsmekanismen. Bells olikhet i sin ursprungliga form kan luras att certifiera ett osäkert system som säkert, och vi avslutar med att ge förslag på möjliga förbättringar. 


\section{Contents}

1 Introduction 1

1.1 History of Cryptography . . . . . . . . . . . . . 1

1.2 Fundamental Principles of Cryptography . . . . . . . . . . . 2

1.3 Public-Key Cryptography . . . . . . . . . . . . . . 5

1.4 Cryptography and the Quantum World . . . . . . . . . 6

1.5 Outline .................. 8

2 Basic Concepts 11

2.1 Linear Algebra . . . . . . . . . . . . . . . . . . 11

2.2 Fundamental Quantum Mechanics . . . . . . . . . . 12

3 A Brief History of QKD 17

3.1 The BB84 Protocol . . . . . . . . . . . . . . . . 18

4 Bell's Theorem 23

4.1 EPR and Hidden Variables . . . . . . . . . . . . . 23

4.2 Bell's Inequality: A Simple Example . . . . . . . . . . . . . . . . 26

4.3 Bell's Theorem . . . . . . . . . . . . . . . . . . 29

4.4 A QKD Protocol Based on Bell's Theorem . . . . . . . . . 31

4.5 Device-Independent QKD . . . . . . . . . . . . . 33

4.6 Loopholes in Bell Experiments . . . . . . . . . . . . 36

5 Energy-Time Entanglement 39

5.1 The Franson Interferometer . . . . . . . . . . . . . . . 40

5.2 Loopholes in the Franson Interferometer . . . . . . . . . . 42

5.3 Realization of Loopholes . . . . . . . . . . . . . . 44

6 Conclusions and Future Work 47 
Publications

A Energy-time entanglement, elements of reality, and local realism 63

B Hacking energy-time entanglement-based systems with classical light 


\section{List of Figures}

1.1 Basic communication scheme for cryptography. Alice and Bob use cryptography to communicate securely in the presence of an eavesdropper, Eve. The message is encrypted using an encryption key, turning the plaintext into a ciphertext and broadcasting it over a public channel. Bob then uses the decryption key to recover the message. . . . . . . . . 4

3.1 The BB84 protocol. This is a prepare-and-measure QKD scheme where Alice encodes information using photons polarized in non-orthogonal bases. Bob chooses from two measurement settings. . . . . . . . . . . . 20

4.1 The EPR-Bohm thought experiment. A $\pi$ meson decays into a positron and electron with opposite spin and these particles are measured by Alice and Bob. . . . . . . . . . . . . . 24

4.2 A simple thought experiment for deriving Bell's inequality. . 27

4.3 The black box method ignores the inner workings of the analysis stations and instead model them as boxes with $N$ push buttons and the outputs +1 and $-1 \ldots \ldots \ldots$

4.4 The E91 setup. A $\pi$ meson decays into two entangled particles that are measured along different axes by Alice and Bob. The resulting correlation violates Bell's inequality.

5.1 The Franson interferometer. The source emits time-correlated photons that are sent to Alice and Bob. At their respective analysis stations, they perform measurements with settings $\phi_{A}$ and $\phi_{B}$ and record the outcome as well as time of detection. 40 
5.2 Schematic of a beam splitter manufactured by joining two triangular prisms with different refractive indices. The beam splitter depicted here has a high refractive index in the lower left region and a low refractive index in the top right. Two incident beams are combined into two output beams. The beam from the left receives no phase shift but the beam from the top receives a $\pi$ phase shift when reflected off the higher refractive index region. . . . . . . . . . . . . 


\section{Preface}

This thesis contains results from research performed by the author at the Information Coding Group at the Department of Electrical Engineering at Linköping Univeristy, Sweden. Parts of the material have been presented at international conferences, and two published or submitted research papers are enclosed at the end of this thesis. 


\section{Acknowledgments}

I would like to express gratitude to my advisor, Jan-Åke Larsson, for his support, patience and encouragement throughout my graduate studies. His guidance has helped me tremendously, and thanks to him I was able to overcome the many hurdles that I encountered in the process of writing this thesis.

My thanks also go to my co-supervisors, Fredrik Karlsson and Peter Stenumgaard for their guidance.

I also want to thank my wife Anna for her understanding, love, and for always standing by my side. Long before I started my graduate studies I knew that I had found an awesome and loyal life companion, and the past years has shown this to be more true than ever.

My colleagues at the Information Coding Group and the Department of Electrical Engineering have made me feel welcome from the first day. Thank you for providing an excellent working atmosphere and for bringing so much fun to the workplace.

I am indebted to all those who have supported me, including Monica, Jan-Erik, and Andreas.

Last but not least I would thank my parents, Eva and Stefan, and my sisters, Susanna and Elisabeth, for their continued support and love.

Jonathan

Linköping, February 2015 


\section{Chapter 1}

\section{Introduction}

This first chapter is intended to give a brief historic overview of how cryptography has evolved from ancient Egypt and Greece all the way to the focus of this thesis; quantum cryptography. This history of increasingly sophisticated cryptographic methods will lead up to our goal of a provably secure cryptographic system. At the same time, codebreakers have been busy refining their methods, and in that spirit we will demonstrate an attack on a seemingly-unbreakable cryptographic system at the end of this thesis.

\subsection{History of Cryptography}

The art of cryptography, or secret writing, appears to be as old as writing itself. The ancient Egyptian civilization left behind documents of hieroglyphs in the Giza pyramids, some of which are believed to be an early example of secret writing. Before the Rosetta stone was discovered, there was no way of understanding the complicated hieroglyphs and therefore the script in itself can be seen as an early example of secret writing. Even with the Rosetta stone, however, there are documents from Giza that still defy translation [1].

From the very beginning, cryptography has put its mark on history by influencing major events and especially wars. In ancient Greece the skytale was used as an early form of transposition cipher. A piece of parchment, cloth or leather is wound around a rod of a certain diameter, and it is then possible to write a message along the length of the rod. When the parchment is unwound, it becomes difficult to comprehend the meaning of the letters that now have moved around, and the recipient can recover the message by winding around a rod of similar diameter. It is believed [1] that the Spartan 
general Lysander used the skytale to secure critical information in a battle against the Persians in $405 \mathrm{BC}$, and the subsequent victory of the Spartans had a lasting impact on early European history. The idea that the skytale was used as a cryptographic device dates back to Cicero (106-43 BC) [2], however this idea has come under scrutiny in recent times. In 1998, after studying the available Greek source material, Kelly [3] claims that "the skytale was nothing more than a piece of leather or parchment attached to a stick" [3, p. 260].

Closely related to cryptography, the field of cryptanalysis concerns itself with analysing cryptographic systems in order to find weaknesses, hidden properties and even break their security. Together with cryptography, the two fields make up the science of cryptology.

In contrast to the many other advances the Chinese civilization managed to achieve, it did not contribute to the development of cryptography as their language lacked a simple alphabet [4]. Instead, it was in the Italian city-states of the renaissance where the first seeds of modern cryptography were sown with clerks writing documents in code. An early example of what we now call a substitution cipher can be found in correspondence from the Vatican some time after the year 1330 [2, p. 280]. Venice and other Italian city-states came to possess some cryptological expertise, and a prime example is the Florentine cryptographer Alberti. Kahn [5, p. 125] describes Alberti as the "Father of Western Cryptology", and his 25-page manuscript De componendis cyfris from 1466/67 is the oldest surviving text on cryptanalysis in the western world [2, p. 280].

\subsection{Fundamental Principles of Cryptography}

The word "cryptography" is constructed from Greek, where kryptós means "hidden" and graphein means "writing". Ever since the renaissance, cryptographers have been in a cat-and-mouse game with cryptanalysts where the former tries to create cryptographic systems that the latter is unable to break while cryptanalysts attempt to mount better and better attacks.

While it is debated whether or not the previously-mentioned skytale was used for cryptography, Herodotus (ca. 486-425 BC) [6] tells the story of a related cryptographic technique. Demeratus, a Greek at the Persian court, sent a secret message by hiding it in a writing tablet. He removed its wax surface and after inscribing a secret message on the wooden backing, he applied a fresh layer of wax which made the tablet appear blank. According 
to Herodotus, the deception was so effective that it fooled not only the Persian customs, but almost the recipient as well.

This method of Demeratus', disguising a message where nobody would look, is called steganography. Not to be confused with the handwriting technique of stenography, there are numerous ways in which steganography has been used throughout history. Invisible ink and microdots are famous examples from spy novels, but there are ways of hiding information in even more plain sight. A digital image can be altered so that the least significant bits constitute a message without the human eye noticing, and a carefully written letter can look innocent while, say, every 21st letter makes up a hidden message. Steganography is one of three basic types of cryptography and truly lives up to the description "hidden message".

The two other basic types of cryptography are codes and ciphers. Codes are used to replace specific words, names or sentences with other words or symbols using a code book, and this method was famously used by Mary, Queen of Scots in a failed attempt to conquer the English throne in the late 16th century [7, pp. 32-44]. Codes and code books are however cumbersome to use, and in modern times the focus has instead shifted towards ciphers. While the definition of a cipher partially overlaps with that of a code, ciphers generally operate on a lower level. The skytale, for instance, is a cipher that operates on individual letters and performs a transposition.

As we have seen in these brief examples, cryptography has historically been used to ensure secrecy when communicating over an untrusted channel. This has changed dramatically with the digital revolution, and new developments in cryptography have led to applications such as authentication, digital signatures, secret sharing and so on. These successes have made technologies like online banking, credit cards, electronic commerce etc. to be secure enough to be appealing to the general public. Cryptography has also led to the development of decentralized cryptographic currencies like Bitcoin [8] that offer an alternative to traditional currencies.

The basic communication scheme for cryptography is depicted in figure 1.1. Two parties, Alice and Bob, wish to communicate securely in the presence of an evesdropper Eve. Alice encrypts her messsage, called the plaintext with a pre-determined encryption algorithm using an encryption key. This turns the plaintext a ciphertext, which is transmitted over an untrusted channel to Bob. During transmission it is assumed that Eve has full knowledge of the ciphertext. Bob decrypts the ciphertext with the decryption key and, if the process is performed correctly, recovers the message.

Before any further analysis of cryptography can be made, however, we 


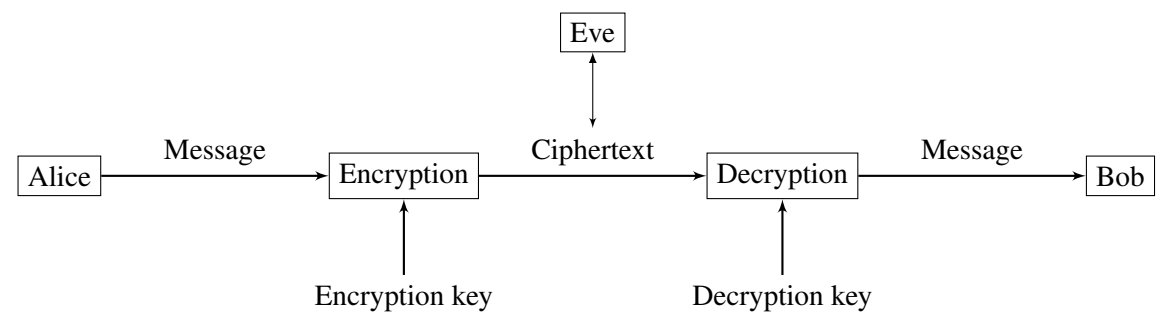

Figure 1.1: Basic communication scheme for cryptography, adapted from [9, p. 3]. Alice and Bob use cryptography to communicate securely in the presence of an eavesdropper, Eve. The message is encrypted using an encryption key, turning the plaintext into a ciphertext and broadcasting it over a public channel. Bob then uses the decryption key to recover the message.

must establish a fundamental principle of cryptology known as Kerckhoff's principle: The enemy knows the system. The importance of this assumption cannot be understated, as the only way to know that a cryptographic system really is secure is if it can withstand the best cryptanalysis. Were Alice and Bob to choose a cryptographic system which in any way relies on Eve not knowing the inner workings of their system, they will probably fool themselves. If Eve happens to learn the trick(s) Alice and Bob have used she will instantly be able to break their security. It is better to let only the key be secret.

In fact, if Alice and Bob invent their own cryptographic algorithms, there is a large probability that their creation will be very insecure. An important principle in cryptography, Schneier's Law, states "Anyone, from the most clueless amateur to the best cryptographer, can create an algorithm that he himself can't break." [10]. Alice and Bob are therefore best advised to rely on methods and algorithms that have been tested and tried by repeated cryptanalysis. The temporary gain that might arise from introducing a secret trick pales in comparison to the permanent damage caused by an unknown flaw in the design*. Our scheme in figure 1.1 must therefore be extended with the assumption that the only thing Eve does not know is the key and the message itself.

\footnotetext{
*In contrast to what many designers of quantum key distribution systems seem to believe, Schneier's law applies to quantum systems, too. It appears that an addendum to Schneier's law is called for: "Any physicist can construct a quantum key distribution system with enough unconditional security that he or she cannot break it."
} 
Cryptographic systems that are in violation of Kerckhoff's principle are said to rely on security through obscurity. It should be obvious that a cryptographic system that in any way relies on steganography is guilty of this flawed security practice.

\subsection{Public-Key Cryptography}

In figure 1.1 there are two keys; one for encryption and one for decryption. Up until the early 1970's, all cryptographic protocols used symmetric algorithms which means that the two keys are identical. The discovery of asymmetric cryptography, or public-key cryptography, revolutionized the field of cryptology by instead using two different keys; one key for encryption and one for decryption. The advantage of public-key cryptography is especially obvious in today's age of the Internet as it does not require Alice and Bob to have met in advance. Leeuw [11, p. 17] writes that public-key cryptography "turned out to be the most important innovation in cryptology since the advent of the computer and it took only a decade to become an indispensable technology for the protection of computer networks".

The first discovery of a public-key algorithm was long credited to the groundbreaking work of Diffie and Hellman in 1976 [12], and their algorithm, Diffie-Hellman (DH) key exchange, allows Alice and Bob to exchange a key over an untrusted channel. It would turn out, however, that DH was not the first invention of its kind. In 1997, the Government Communications Headquarters (GCHQ) in the United Kingdom declassified information that revealed a similar discovery made several years earlier [7, pp. 283-290]. Due to the secret nature of intelligence work, the original inventors at GCHQ had to wait over two decades before their achievement was recognized publicly. The original motivation for the research that led up to this discovery by the GCHQ was to reducing the cost of distributing symmetric keys [7, p. 282].

Public-key cryptography can be created from a special type of mathematical functions that are one-way. This is a function $f$ with the property that, given $x$, computing $y=f(x)$ is easy while it is computationally infeasible to find $x$ so that $f(x)=y$. If the one-way function also has a trapdoor it means that there exists a way to find $x$, but only with some extra information that is known only to the designer of said function. It should be computationally infeasible for someone else to determine this trapdoor information [9, p. 191]. Trapdoor one-way functions allow us to create algorithms for public-key cryptography. 
From a very large family of such functions, Bob generates one in such a way that only he knows the corresponding trapdoor information. He then publishes his function $f$ as his public encryption algorithm. Alice, who wants to send Bob the message $m$, computes the ciphertext $c=f(m)$ and sends this to Bob. He can then compute the message $m$ using the trapdoor information, but Eve can not. Using an one-way trapdoor function we now create a public-key cryptosystem where Alice and Bob can communicate securely without a pre-shared key.

The one-way trapdoor function used in DH is modular exponentiation [12], and in order to reverse the trapdoor one needs to solve the discrete logarithm problem which is considered hard. The GCHQ public-key algorithm, however, uses a different one-way trapdoor function based on the factorization problem. Computing $f(m)=m^{e}(\bmod n)$ is easy given $e$ and $n$, but without knowing the prime factors $p$ and $q$ of $n$ (the trapdoor information), the reverse is computationally infeasible for large $n$. The same method was re-discovered by Rivest, Shamir, and Adleman independently of GCHQ and is named RSA [13] after the inventors. RSA remains the most popular public-key algorithm of today [14, p. 17].

It must be pointed out that there is no proof that the discrete logarithm problem or the factoring problem is difficult. It is theoretically possible that, however unlikely, there will be a major breakthrough tomorrow that allows for instantaneous factorization, which would break RSA. However, the peculiar properties of prime numbers have been studied since at least Euclid's time (300 BC) and it is likely that computing prime factors will remain difficult.

\subsection{Cryptography and the Quantum World}

Research into the factorization problem took an unexpected turn in 1994, when Shor [15] published an efficient quantum algorithm for finding prime factors. The difference to previous factoring algorithm is that Shor's algorithm requires a quantum computer, a device operating on qubits instead of ordinary, classical bits. The RSA algorithm typically uses prime factors hundreds of digits long, but the current record in quantum computing is from 2012, when Martín-López et al. [16] factored the number 21 into its prime factors 3 and 7. In the near future, Shor's algorithm remains a theoretical rather than practical threat, however the mere idea of a quantum computer has led researchers to search for algorithms that remain strong even if a 
revolution in quantum computing would occur.

This relatively new area of research is called post-quantum cryptography and aims to identify weak parts of current cryptographic algorithms and replace them with those safe from quantum computers. In fact, several systems used today are already secure [17, pp. 1-2] and although most are symmetric algorithms, McEliece's Goppa code [18] is an example of a post-quantum public-key algorithm. This system, however, has a rather low efficiency as it requires several orders of magnitude more key bits to reach the same strength as RSA [17, p. 3].

We will now turn our attention to a cryptosystem that achieves security without resorting to not-yet-proven assumptions on a problem being difficult. The one-time pad (OTP) has unconditional security [14, pp. 15-17, 9, pp. 39-41] and no matter what computing power Eve possesses she will not be able to break it. The OTP has been described as "the Holy Grail of cryptography" [7, p. 122], but the disadvantage is that it is a symmetric scheme that puts enormous demand on key management. For every bit of information that is to be encrypted, one bit of key is needed. Add to it the fact that the key must be random, secret and never re-used this costly scheme has primarily been used in low-bandwidth applications with ultra-high security requirements [14, p. 17].

If Alice and Bob want to base their security on the OTP and transfer, say, a gigabyte of information, they will need a gigabyte of key. If their key runs out, they cannot reuse any part of it and will have to negotiate more key bits. It is, of course, possible to use a public-key algorithm to generate such a key, but the chain cannot be stronger than the weakest link and this would be a pointless implementation of the OTP. As it stands, Alice and Bob will have to rely on a trusted courier to exchange keys and let him or her carry the entire burden of securing their communication.

In the classical world this is as good as it gets. The OTP gives ultimate security, but shifts the entire problem of encryption into a problem of key management. There is simply no way around it; Alice and Bob must meet in person or use a courier. Unless, of course, they again turn to invoke quantum mechanics. The peculiar properties of a quantum channel allows Alice and Bob to set up a communications system where the laws of physics, not vague concepts of "computational complexity", guarantee the security. These same laws also makes the system robust against an attacker who uses a working quantum computer.

The idea is to use the quantum channel in such a way that Alice and Bob generate a secret key to be used in an OTP. The result is quantum key 
distribution (QKD) and this key distribution method can give perfect security. QKD is a field currently undergoing tremendous development and there are several working protocols, such as the original BB84 protocol by Bennett and Brassard [19], E91 [20] by Ekert, Coherent One-Way (COW) [21] and Distributed Phase-Shift (DPS) [22].

Recently, research into so-called energy-time entanglement has begun leading the way into a new and robust method to apply quantum mechanics to cryptography. It has been suggested that a design by Franson [23] could be used to achieve the same unconditional security as traditional QKD protocols. Several experiments have evaluated this Franson-type setup [2431], however this thesis will show that there are complications when basing QKD on energy-time phenomena.

\subsection{Outline}

This thesis will present our contributions in energy-time entangled quantum key distribution in publications $\mathrm{A}$ and $\mathrm{B}$ enclosed at the end of this thesis. The chapters leading up to these two papers are intended to give an overview of the field of quantum key distribution and highlight the important theory of Bell's theorem.

We begin in chapter 2 by establishing notation, followed by some brief notes on linear algebra. This theory is then used to discuss a few basic postulates in quantum theory that will have important consequences for quantum key distribution, and we then build on these postulates to prove the important theorems of no-cloning and non-distinguishability of nonorthogonal quantum states.

Chapter 3 surveys the field of quantum key distribution and presents two major categories of protocols; those that are of the type prepare-and-measure, and those that are based on entanglement. We then present the pioneering BB84 protocol that is built on non-orthogonal polarization of individual photons.

Our contributions in publications A and B rely heavily on Bell's theorem and we therefore dedicate chapter 4 to discussing some of the consequences of this fundamental limitation imposed by Nature herself. We also show how the theorem leads to the quantum-mechanical phenomenon of entanglement. Loopholes in Bell tests, presented in section 4.6, show us that we must make sure to understand and quantify the amount by which experiments deviate from the ideal situation. 
Energy-time entanglement, the focus of our research, is introduced in chapter 5 and we show what advantages this method has over traditional, polarization-based QKD. In the same chapter, the Franson interferometer is presented in detail. At this point we have all the necessary background to show how energy-time-entangled systems can be compromised.

Our main contributions are publications A and B. There, we show how the postselection of the Franson interferometer can be exploited by an attacker to gain full control of the system. Our attack is tailored to be invisible to Alice and Bob even in the presence of noise, and we conclude by suggesting countermeasures and alternative setups.

We conclude in chapter 6 by returning to the bigger picture and what needs to be done to make QKD a truly unbreakable system. We also present ideas for future work. 


\section{Chapter 2}

\section{Basic Concepts}

Ever since the foundations of cryptography were formulated in mathematical terms, cryptology and cryptanalysis has been a game largely played within the field of mathematics. Quantum mechanics, whose laws have been discovered through experiment and theory, has led to significant developments in modern society. In order to understand QKD, one needs to know its mathematical foundations and how they apply to our purposes of securing communications. This chapter will present the notation used in the rest of the thesis followed by important concepts in linear algebra. Then we move on to discuss a few essential postulates of quantum theory and their implications.

\subsection{Linear Algebra}

Linear algebra is used in many applied fields, and the wide variety of flavors has led different authors to adapt conflicting standards to how concepts translate into notation. For the rest of this thesis we will work with the vector space $\mathbb{C}^{n}$. A vector within this space is written $|\phi\rangle$, where the $\phi$ is the actual label of our vector. The entire object is called a ket, and its vector dual is the bra $\langle\phi|$. This useful Bra-ket notation was invented by Dirac [32].

The complex conjugate of the number $z$ is written $z^{*}$. Similarly, the element-wise complex conjugate of a matrix $A$ is $A^{*}$. The identity matrix is denoted $I$, the transpose of a matrix $A$ is $A^{T}$ and the Hermitian conjugate is $A^{\dagger}=\left(A^{T}\right)^{*}$. Given a vector $|\phi\rangle$ its vector dual $\langle\phi|$ can be computed as the Hermitian conjugate $\langle\phi|=(|\phi\rangle)^{\dagger}$.

The inner product is a function that takes two vectors on a Hilbert space $\mathscr{H}$ and produces a complex number. We write this as $(\cdot, \cdot): \mathscr{H} \times \mathscr{H}$ to $\mathbb{C}$. 
On $\mathbb{C}^{n}$ the inner product of the vectors $\mathbf{x}=\left(x_{1}, \ldots, x_{n}\right)$ and $\mathbf{y}=\left(y_{1}, \ldots y_{n}\right)$ is defined by vector multiplication:

$$
\left(\left(x_{1}, \ldots, x_{n}\right),\left(y_{1}, \ldots y_{n}\right)\right)=\left[\begin{array}{lll}
x_{1}^{*} & \ldots & x_{n}^{*}
\end{array}\right]\left[\begin{array}{c}
y_{1} \\
\vdots \\
y_{n}
\end{array}\right] .
$$

We can write the inner product of two vectors $\left|\phi_{1}\right\rangle$ and $\left|\phi_{2}\right\rangle$ as $\left\langle\phi_{1} \mid \phi_{2}\right\rangle$. Two vectors $\left|\phi_{1}\right\rangle$ and $\left|\phi_{2}\right\rangle$ are said to be orthogonal if their inner product is zero.

Of particular interest is a class of maps that are unitary, that is, their matrix representations fulfill

$$
U U^{\dagger}=U^{\dagger} U=I
$$

Later, in Postulate 3 we will see that unitary matrices play an important role in quantum mechanics.

Remark 1 An important property we will use later on is the fact that the inner product is invariant under unitary transformation. Let $\left|\phi_{1}\right\rangle$ and $\left|\phi_{2}\right\rangle$ be two vectors in a Hilbert space. Then

$$
\left(U\left|\phi_{1}\right\rangle, U\left|\phi_{2}\right\rangle\right)=\left\langle\phi_{1}\left|U^{\dagger} U\right| \phi_{2}\right\rangle=\left\langle\phi_{1}|I| \phi_{2}\right\rangle=\left\langle\phi_{1} \mid \phi_{2}\right\rangle .
$$

\subsection{Fundamental Quantum Mechanics}

Now that we have established our notation for linear algebra we move on to briefly define some of the postulates of quantum mechanics. For a more complete description, see the textbook by Nielsen and Chuang [33].

Postulate 1 An isolated physical system is associated with a Hilbert space $\mathscr{H}$, known as the state space. The system is completely described by its state vector which is a unit vector in the system's state space.

The state vector is usually written as the ket vector $|\phi\rangle$. A quantum system where the state is fully known is said to be in a pure state.

Note that Postulate 1 only deals with isolated systems. If the universe only consisted of isolated systems it would be a very dull place, so we need a way for them to interact. Therefore, the next postulate has to do with measurement: 
Postulate 2 A collection of measurement operators $\left\{M_{m}\right\}$ operating on the state space make up a quantum measurement. The index $m$ refers to the possible measurement outcomes, and the probability of observing outcome $m$ from a system with state $|\phi\rangle$ is

$$
p(m)=\left\langle\phi\left|M_{m}^{\dagger} M_{m}\right| \phi\right\rangle .
$$

We can put together several quantum systems by using the tensor product on their state vectors. The tensor product of the systems $\left|\phi_{1}\right\rangle$ and $\left|\phi_{2}\right\rangle$ is written

$$
\left|\phi_{1}\right\rangle \otimes\left|\phi_{2}\right\rangle=\left|\phi_{1}\right\rangle\left|\phi_{2}\right\rangle=\left|\phi_{1} \phi_{2}\right\rangle,
$$

and we will frequently make use of this shorthand notation.

Finally we deal with how quantum systems evolve over time and use the previously mentioned unitary maps:

Postulate 3 An isolated quantum system evolves over time by unitary transformation. If the state of a system at a given point in time is $|\phi\rangle$, the state at a later time is $U|\phi\rangle$ where $U$ is an unitary matrix that only depends on the start and end times.

In the classical world we are used to being able to use measurements to determine which state a system was in. We can, for instance, identify on which side a coin has landed. The quantum world is not as simple. If we have a collection of possible states $\left\{\phi_{i}\right\}$ and want to determine which of the state we actually are dealing with, we can only do this reliably when the states are orthogonal. To see how orthogonal states are distinguished, we simply define the measurement operators* $M_{i}:=\left|\phi_{i}\right\rangle\left\langle\phi_{i}\right|$ for all $i$. We can now see that a state $\left|\phi_{i}\right\rangle$ gives the measurement outcome $i$ with probability $p(i)=\left\langle\phi_{i}\left|M_{i}\right| \phi_{i}\right\rangle=1$. Therefore, we can distinguish between orthogonal states with certainty. However, if they are not orthogonal this will not be possible:

Theorem 1 Non-orthogonal states cannot be reliably distinguished

Proof Proof by contradiction. Suppose $\left|\phi_{1}\right\rangle$ and $\left|\phi_{2}\right\rangle$ are not orthogonal and that it is possible to distinguish between them. When distinguishing between these states we perform attempt a quantum measurement $\left\{M_{m}\right\}$ and get an outcome $j$. We then we use some rule $f$ so that, when the state $\left|\phi_{1}\right\rangle$

*We also need to define an operator $M_{0}$ so that the completeness relation is fulfilled, but we skip this part for brevity. 
was prepared, the probability of measuring $j$ so that $f(j)=1$ is 1 . Similarly, when $\left|\phi_{2}\right\rangle$ was prepared, we have unity probability of measuring $j$ so that $f(j)=2$. Now define the quantity

$$
E_{i}:=\sum_{j: f(j)=i} M_{j}^{\dagger} M_{j}
$$

and rewrite the rule function $f$ as

$$
\left\langle\phi_{1}\left|E_{1}\right| \phi_{1}\right\rangle=1 ; \quad\left\langle\phi_{2}\left|E_{2}\right| \phi_{2}\right\rangle=1 .
$$

Using $\sum_{i} E_{i}=I$ we see that $\sum_{i}\left\langle\phi_{1}\left|E_{i}\right| \phi_{1}\right\rangle=1$, and together with equation (2.7) we see that $\left\langle\phi_{1}\left|E_{2}\right| \phi_{1}\right\rangle=0$ which gives $\sqrt{E_{2}}\left|\phi_{1}\right\rangle=0$. We now rewrite $\left|\phi_{1}\right\rangle$ as a linear combination of $\left|\phi_{2}\right\rangle$ and some other state vector $|\psi\rangle$ orthonormal to $\left|\phi_{1}\right\rangle$. We know that $|\alpha|^{2}+|\beta|^{2}=1$, and since $\left|\phi_{1}\right\rangle$ is not orthogonal to $\left|\phi_{2}\right\rangle$, we have $\beta<1$. We now show the following inequality:

$$
\left\langle\psi\left|E_{2}\right| \psi\right\rangle \leq \sum_{i}\left\langle\psi\left|E_{2}\right| \psi\right\rangle=\langle\psi|| \psi\rangle=1,
$$

and since $\sqrt{E_{2}}\left|\phi_{2}\right\rangle=\beta \sqrt{E_{2}}|\psi\rangle$ we get

$$
\left\langle\phi_{2}\left|E_{2}\right| \phi_{2}\right\rangle=|\beta|^{2}\left\langle\psi\left|E_{2}\right| \psi\right\rangle \leq|\beta|^{2}<1 .
$$

Note now that this is in contradiction with equation (2.7) which states that the probability must be 1 . Therefore, the assumption that $\left|\phi_{1}\right\rangle$ and $\left|\phi_{2}\right\rangle$ can be reliably distinguished is false.

We see that when non-orthogonal states are measured, there will be a nonzero probability of error. Another important feature of quantum mechanics is the no-cloning theorem:

Theorem 2 It is impossible to make a copy of an unknown quantum state.

We prove this theorem in the same way as we proved theorem 1; by contradiction. We will only discuss the case of unitary evolution, and for a discussion of non-unitary evolution and the no-cloning theorem, see Nielsen and Chuang [33, p. 532].

Proof Assume that cloning is possible. We can then build a quantum machine that performs quantum cloning which has one input and one output slot. We put an unknown state $|\phi\rangle$ in the input slot and the machine copies 
this state into the output slot. The output slot of the machine is in some state $|s\rangle$ just before the cloning process starts. We write this as

$$
|\phi\rangle \otimes|s\rangle .
$$

We now let this system evolve unitarily according to Postulate 3 which gives us

$$
U(|\phi\rangle \otimes|s\rangle)=|\phi\rangle \otimes|\phi\rangle .
$$

In particular, we assume that this general machine copies two pure states: $|\phi\rangle$ and $|\psi\rangle$ :

$$
\begin{aligned}
& U(|\phi\rangle \otimes|s\rangle)=|\phi\rangle \otimes|\phi\rangle \\
& U(|\psi\rangle \otimes|s\rangle)=|\psi\rangle \otimes|\psi\rangle .
\end{aligned}
$$

From remark 1 we know that the inner product is invariant under unitary transformation, so the inner product of these two equations they will be equal. This gives

$$
\langle\phi \mid \psi\rangle=(\langle\phi \mid \psi\rangle)^{2},
$$

and the only solutions in $\mathbb{C}$ are $\langle\phi \mid \psi\rangle=0$ and $\langle\phi \mid \psi\rangle=1$. Since a state vector always is of length 1 we see that the only time it is possible to clone an unknown quantum state is when they are equal or orthogonal.

Quantum mechanics therefore only allows measurement outcomes to be copied, not general states. This will be important for a QKD system since Eve will not be able to make a copy of an unknown state. 


\section{Chapter 3}

\section{A Brief History of QKD}

One fine afternoon in late October 1979, I was swimming at the beach of a posh hotel in San Juan, Puerto Rico. Imagine my surprise when this complete stranger swims up to me and starts telling me, without apparent provocation on my part, how to use quantum mechanics to design unforgeable banknotes! This was probably the most bizarre, and certainly the most magical, moment in my professional life. [...] Thus was born a wonderful collaboration that was to spin out [...] quantum cryptography. (Gilles Brassard, 2005 [34])

As with many other advances in science, the discovery of QKD with a good story. Five years before they would publish their seminal paper [19], Bennett and Brassard met while swimming in the Atlantic Ocean outside of San Juan. They started thinking about encoding information sent between Alice and Bob as polarized photons, which would make it difficult for Eve to intercept their message since a quantum system cannot be measured without introducing noise. They realized that this noise would then be detected by Alice and Bob, and they could take appropriate actions to protect their transmission. This mechanism of using quantum mechanics to transfer information could be used to generate a key for an OTP session.

As mentioned in chapter 1, OTP is as secure as its method for key distribution. Now follows the motivation for quantum key distribution in general and this thesis in particular: A quantum system together with OTP can provide Alice and Bob with a provably secure means of communication without having to agree on a key in person. With a quantum channel (for instance, polarized photons sent through an optical fiber) they can instead 
generate the key in real time for as long as they want.

We will discuss QKD protocols involving two parties, Alice and Bob, who want to communicate without leaking information to Eve. The term "interferometer" is used in quantum mechanics with a wide variety of meanings, however in this thesis its usage will be restricted to mean an entire QKD device, and not any of its components. The devices Alice and Bob use for measurement and detection are called analysis stations.

In addition to the quantum channel, Alice and Bob need an authenticated classical channel to discuss basis choices after the quantum transmission is complete. This channel is public and any transmission made here is assumed to be known to Eve.

The QKD protocols discussed in this thesis fall into two general categories, prepare-and-measure and entanglement-based. In a prepare-andmeasurement scheme, Alice prepares a quantum state and sends it to Bob who then makes an appropriate measurement. The entanglement-based protocol is slightly more complicated than the prepare-and-measure setup. In addition to Alice's and Bob's analysis stations there is a source device responsible for generating the quantum states. Some examples of entanglement-based protocols is Ekert's E91 and designs based on the Franson interferometer (see section 5.1).

In both the prepare-and-measure and entanglement-based setup, the analysis stations can be modelled as a device with the quantum channel as input, $N$ push buttons as local setting and output of either 0 (no detection), +1 (plus detection) or -1 (minus detection). In mathematical terms the setting can take on the values $1, \ldots, N$ and we denote Alice's analysis station as the function $A:\{1, \ldots, N\} \rightarrow\{-1,0,+1\}$. Bob's analysis station is defined as the function $B$ in the same way. In the general case, the output of the analysis station might depend on not only the local, but also the remote setting.

If the analysis station returns 0 as measurement output it means that no event was detected at all, and whenever there is no ambiguity we will use the shorthand notation

$$
A_{i}:=A\left(\phi_{A_{i}}\right) \quad \text { and } \quad B_{j}:=B\left(\phi_{B_{j}}\right) .
$$

\subsection{The BB84 Protocol}

The first QKD protocol to be discovered was BB84, named after the inventors Bennett and Brassard and the year it was first published in print, 1984 [34]. $\mathrm{BB} 84$ is a protocol based on the prepare-and-measure principle, and the 
qubits are individual polarized photons. The following description of the protocol is adapted from the texbook of Nielsen and Chuang [33, pp. 587588].

In the preparation phase, Alice prepares polarized photons, each polarized along an angle randomly choosen from the four following directions: horizontal $(-)$, vertical $(\mid),+45^{\circ}$ diagonal $(~ /)$ and $-45^{\circ}$ diagonal $(\backslash)$. She sends these photons to Bob, who makes a polarization measurement either in the rectilinear basis $(+)$ or the diagonal basis $(\times)$. Now begins the measurement phase. For each incoming photon, Bob randomly chooses between these two methods and remembers both the measurement outcome and which measurement was performed.

We express Alice's four base state in the bra-ket notation as

$$
\begin{aligned}
& \left|\psi_{00}\right\rangle=|0\rangle \\
& \left|\psi_{10}\right\rangle=|1\rangle \\
& \left|\psi_{01}\right\rangle=|+\rangle=\frac{1}{\sqrt{2}}(|0\rangle+|1\rangle) \\
& \left|\psi_{11}\right\rangle=|-\rangle=\frac{1}{\sqrt{2}}(|0\rangle-|1\rangle) .
\end{aligned}
$$

The first subscript of $|\psi\rangle$ is the bit value, and the second subscript represents the basis in which the photon is polarized. The bases in this case are two of the Pauli matrices

$$
\begin{aligned}
\sigma_{x} & =\left(\begin{array}{cc}
0 & 1 \\
1 & 0
\end{array}\right) \\
\sigma_{z} & =\left(\begin{array}{cc}
1 & 0 \\
0 & -1
\end{array}\right) .
\end{aligned}
$$

Note that these four states are not orthogonal, and therefore cannot be distinguished by quantum measurement as seen in theorem 1 . Let $\delta \geq 0$ be a natural number. Alice now uses her random number generator (RNG) to generate two bit strings $a$ and $b$, each of length $(4+\delta) n$. The values of these strings are kept secret and are used to create the quantum state

$$
|\psi\rangle=\bigotimes_{k=1}^{(4+\delta) n}\left|\psi_{a_{k} b_{k}}\right\rangle,
$$

where the subscript $k$ is the $k^{\text {th }}$ bit of the strings. The resulting state $|\psi\rangle$ is the tensor product of the base states in equation (3.2). Now Alice sends 


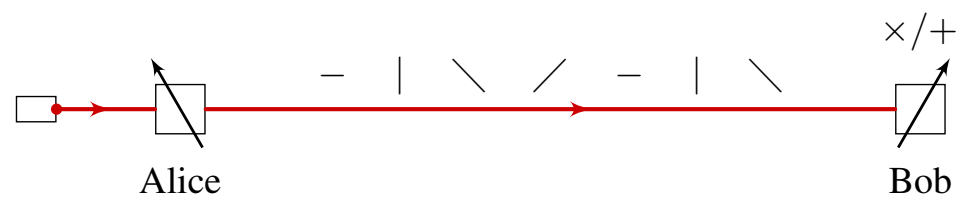

Figure 3.1: The BB84 protocol. This is a prepare-and-measure QKD scheme where Alice encodes information using photons polarized in non-orthogonal bases. Bob chooses from two measurement settings.

$|\psi\rangle$ over an untrusted quantum channel to Bob. He will receive the state, possibly affected by noise and Eve, announces this to Alice over the public channel, and randomly generates a bit string $b^{\prime}$ of his own, again of length $(4+\delta) n$.

Bob now performs quantum measurements on his received state according to these random bits. If the bit value is 0 , he measures in the basis $\sigma_{X}$ and if it is 1 he measures in $\sigma_{Z}$. The measurement result will be either 0 or 1 , and Bob stores this data in a new bit string $a^{\prime}$. At this point, Alice and Bob have gathered what is called the raw key. This raw key needs to be processed and analyzed in several steps before it can be used. Bob tells Alice over a public channel that he has performed his quantum measurements, and Alice then broadcasts her basis choice $b$.

It might seem peculiar that Alice broadcasts her measurement settings. Could this not be used by Eve to gather the raw key? The answer is no, since the quantum state was forever altered by Bob when he performed his random measurement. Also, Eve cannot hijack the information sent over the quantum channel as we proved in theorem 2 that it is impossible to copy an unknown quantum state.

After Alice broadcasts her choice, she and Bob discard all bits in $a$ and $a^{\prime}$ at positions where the bit strings $b$ and $b^{\prime}$ are different. The result is the sifted key which, in theory, could be used to perform OTP. However, it is possible that Eve has performed an attack on the channel, and the next step will tell if this is the case. Again following the reasoning of Nielsen and Chuang [33, pp. 587-588], we assume that $\delta$ is sufficiently large so that the sifted key is at least $2 n$ bits long with high probability.

From these $2 n$ bits, Alice randomly selects a subset of size $n$ and broadcasts the bit positions and values to Bob. He can then compare these check bits with the ones in his sifted key, and as the quantum channel introduced noise during the transmission process, some of the measurement results will 
be noisy. An attempted attack, however, also manifests itself as random noise, and if the error rate is too high the protocol must be aborted. The error threshold is determined so that the two last steps in the protocol, information reconciliation and privacy amplification, can obtain enough secret bits for the subsequent data transfer. A detailed analysis of these two last steps is beyond the scope of this thesis, but it can be shown [33, p. 602] that unconditional security can be achieved if the error rate is below $11 \%$. As long as the noise stays below this limit, Alice and Bob can use the BB84 protocol and be sure that no eavesdropper is present. The secret key they receive from the process is then used to encrypt their message using OTP.

The BB84 protocol thus allows Alice and Bob to agree on a secret key. Any attempt by Eve to eavesdrop will be noticed by Alice and Bob when comparing check bits who will then abort the transmission. There is no way for Eve to learn the value of the key, and due to the provable security of the OTP, the QKD session is perfectly secure. Of course, Eve could perform a denial-of-service attack by cutting or sabotaging the quantum channel, but this will not give her any information about the message.

This concludes our short introduction of the BB84 protocol. Again, this is a prepare-and-measure setup, so Alice and Bob must establish a hierarchy of who is the sender and who is the receiver. The situation will be different in the entanglement-based protocols such as E91 (shown in section 4.4) and Franson-based systems shown in section 5.1. These two new protocols instead rely on violation of the Bell inequality as a security test, and this concept will be discussed in the next chapter. 


\section{Chapter 4}

\section{Bell's Theorem}

Bell's theorem [35] is of considerable importance when trying to understand the very fundamentals of quantum mechanics. The theorem has consequences not only for physics, but also leads to consequences for philosophical interpretations of reality. The ideas presented in this chapter do in some sense go against human intuition because we will have to abandon the ideas of "locality" and "realism". In 1975, Stapp [36, p. 271] claimed that "Bell's theorem is the most profound discovery of science".

\subsection{EPR and Hidden Variables}

In 1935, the early days of quantum mechanics, Einstein, Podolsky, and Rosen (EPR) published a paper [37] where they asked if quantum mechanics could be considered complete. In the paper, they started with a few basic assumptions and used the laws of quantum mechanics to produce an apparent contradiction. The argument, which will be presented now, is sometimes referred to as the "EPR paradox".

Here, we show a later modification of the EPR paradox described by Bohm [38] in 1951. We begin with the so-called Bell state defined as follows:

$$
\frac{1}{\sqrt{2}}(|01\rangle-|10\rangle) \text {. }
$$

Again, $|01\rangle$ is the tensor product of the states $|0\rangle$ and $|1\rangle$. The Bell state can be realized in several ways, but for the purposes of this example we will consider $\pi$ meson decay. The $\pi$ meson (also called pion) is a subatomic 


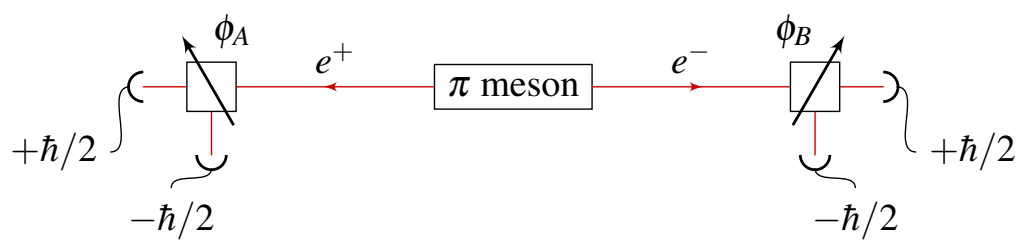

Figure 4.1: The EPR-Bohm thought experiment. A $\pi$ meson decays into a positron and electron with opposite spin and these particles are measured by Alice and Bob.

particle that can decay in several ways. One such way is

$$
\pi^{0} \rightarrow \gamma+e^{-}+e^{+}
$$

where the decay products are one gamma photon, one electron and one positron. If we require the $\pi$ meson to be at rest it will have zero angular momentum and according to the law of conversation of angular momentum, the sum of the angular momenta of the particles on the left-hand side of equation (4.2) must be zero as well. A photon has zero angular momentum and therefore the electron and positron will have opposite spin. These particles, whose intrinsic spin always takes on the values $\pm \hbar / 2$, will have two possible configurations: one where the positive spin component is given to the positron and one where it is given to the electron. We write this as $e^{+} \uparrow$ and $e^{-} \downarrow$, or conversely, $e^{+} \downarrow$ and $e^{-} \uparrow$. The basis states are then $|0\rangle:=|\uparrow\rangle$ and $|1\rangle:=|\downarrow\rangle$ and the system will be in the state

$$
\frac{1}{\sqrt{2}}\left(|0\rangle_{e^{-}} \otimes|1\rangle_{e^{+}}-|1\rangle_{e^{-}} \otimes|0\rangle_{e^{+}}\right)
$$

which can be simplified to the Bell state in equation (4.1).

Now EPR present their argument. Let the electron-positron pair carefully move very far away from each other in a way that retains their angular momentum. Alice receives the positron and Bob the electron, and if Alice performs a spin measurement along the $z$ axis she will get either the result $+\hbar / 2$ or $-\hbar / 2$. Spin can be measured along different axes, and according to Heisenberg's uncertainty principle [39] the spin along orthogonal axes cannot be determined with certainty. If we simultaneously let Bob measure the electron spin along $x$ he will know not only the spin of his own particle but can predict the corresponding value of Alice's positron as well. EPR now argue that the spin along both the $x$ and $z$ axes must exist simultaneously 
in conflict with the uncertainty principle, otherwise measurements on the electron would disturb the positron even though there is no connection between the two particles.

Therefore, according to EPR, one of the following must be true:

1. The particles must be exchanging information faster than the speed of light, or

2. The behavior of the particles is predetermined by some "hidden variables".

The first possibility of instantaneous information exchange was rejected by EPR as it would violate the "principle of locality". In a later paper, Einstein wrote [40]

The following idea characterises the relative independence of objects far apart in space, A and B: external influence on A has no direct influence on $B$; this is known as the Principle of [Locality] ${ }^{*}$, which is used consistently only in field theory. If this axiom were to be completely abolished, the idea of the existence of quasienclosed systems, and thereby the postulation of laws which can be checked empirically in the accepted sense, would become impossible.

(Einstein [40])

EPR therefore concluded that the principle of locality should apply to their thought experiment and therefore rejected the idea of "spooky action at a distance" [37]. The logical consequence of this line of thought is that quantum mechanics is somehow incomplete, since the state vectors do not give a complete description of the individual particles. This is in violation of Postulate 1. Therefore, according to EPR, we must invoke hidden variables, defined by Baggott as follows:

Any theory which rationalizes the behaviour of a system in terms of parameters that are for some reason inaccessible to experiment is a hidden variable theory. ([41, p. 107])

Hidden variable theories have had success in the history of science. For instance, the relation between the volume, pressure and temperature of a gas is very complicated, but when taking the individual atoms into account

*Einstein originally referred to the "Principle of Local Action", however this thesis uses the term "Principle of Locality". 
these properties emerge naturally. In the case of the gas, the atoms are the hidden variables, and measuring individual atoms is very difficult even today. Assuming that hidden variables were responsible for a seemingly complex phenomenon was therefore not a big logical step for EPR to take.

The existence of hidden variables could imply the existence of deeper, more fundamental laws of physics than quantum mechanics. According to Baggott [41], Einstein had "hinted at a statisical interpretation" in a similar spirit to the emergent gas properties that we just described. Perhaps such a theory would allow for a universe where outcomes are deterministic in contrast to the quantum-mechanical laws? In any case, hidden variables are a problem for our goals of secure QKD since we then would be unable to trust our results from theorems 1 and 2 .

\subsection{Bell's Inequality: A Simple Example}

Anyone who is not shocked by quantum theory has not understood it.

(Bohr [42])

No real solution to the EPR paradox was brought up in the decades following the EPR paper. It took until 1964 when Bell published his celebrated theorem [35] that puts limits on what correlations can be achieved by hidden-variable theories. Bell's contribution was to show that that Nature experimentally invalidates EPR's view of the world [33, p. 114]. We will now present another thought experiment, the second so far in this chapter. This one will show how quantum mechanics goes against "common sense" and is adapted from the textbook by Nielsen and Chuang [33, pp. 114-117].

Similar to the EPR experiment in section 4.1, Alice and Bob each have an analysis station that receives particles from a source. The source prepares pairs of particles and sends one to Alice and one to Bob. Alice has a choice of two settings for her analysis station, either measure the (invented) properties $Q$ or $R$ while Bob can measure the (invented) properties $S$ or $T$. The outcomes from the analysis stations are \pm 1 , and the choice between the two settings is determined at random when the particles are received, not before. We let Alice and Bob be far away from each other and arrange the timing so that the measurements are performed simultaneously. Since physical influences cannot propagate faster than light, Alice's measurements cannot influence those made by Bob. The experiment is depicted in figure 4.2.

Continuing the thought experiment, we write down the quantity $Q S+$ 


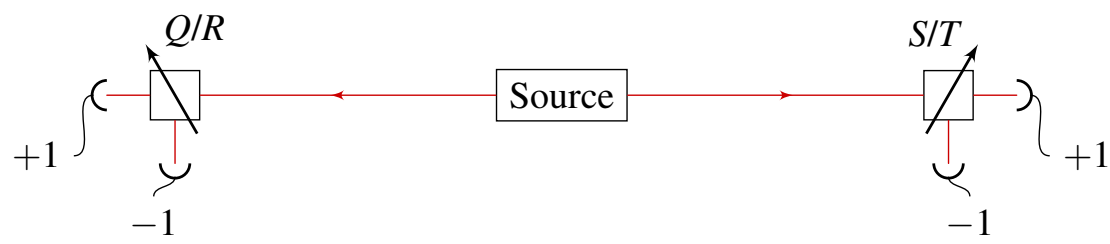

Figure 4.2: A simple thought experiment for deriving Bell's inequality.

$R S+R T-Q T$ and perform some algebraic manipulations:

$$
Q S+R S+R T-Q T=(Q+R) S+(R-Q) T
$$

and since all quantities have magnitude 1 it follows that either $(Q+R) S$ or $(R-Q) T$ equals zero. Therefore, the quantity in equation (4.4) has magnitude 2 . If we also let $p(q, r, s, t)$ represent the probability of the particles being in the state $Q=q, R=r, S=s$, and $T=t$ before being measured, we calculate the expected value of equation (4.4):

$$
\begin{aligned}
E(Q S+R S+R T-Q T) & =\Sigma_{q r s t} p(q, r, s, t)(q s+r s+r t-q t) \\
& \leq 2 \Sigma_{q r s t} p(q, r, s, t) \\
& =2 .
\end{aligned}
$$

At the same time we note

$$
\begin{aligned}
E(Q S+R S+R T-Q T) & =\Sigma_{q r s t} p(q, r, s, t)(q s+r s+r t-q t) \\
& =\Sigma_{q r s t} p(q, r, s, t) q s+\Sigma_{q r s t} p(q, r, s, t) r s \\
& +\Sigma_{q r s t} p(q, r, s, t) r t-\Sigma_{q r s t} p(q, r, s, t) q t \\
& =E(Q S)+E(R S)+E(R T)-E(Q T),
\end{aligned}
$$

and if we put equation (4.6) and equation (4.6) together we obtain the Bell inequality

$$
E(Q S)+E(R S)+E(R T)-E(Q T) \leq 2 .
$$

Inequality (4.7) is a limit on the correlations obtained from a "commonsense" system. Now we ask ourselves what predictions a quantum-mechanical system would give. In this quantum experiment (also depicted in figure 4.2) the source does not prepare classical particles, but qubits. These qubits are in the Bell state defined in equation (4.1) and just like before, one qubit goes to Alice and one to Bob. We now define Alice's and Bob's measurement 
operators in terms of the Pauli matrices introduced in equation (3.3)

$$
\begin{aligned}
Q & =\sigma_{Z} \\
R & =\sigma_{X} \\
S & =-\frac{1}{\sqrt{2}}\left(\sigma_{Z}+\sigma_{X}\right) \\
T & =\frac{1}{\sqrt{2}}\left(\sigma_{Z}+\sigma_{X}\right),
\end{aligned}
$$

and calculate the quantum expectations

$$
\begin{array}{ll}
E_{Q M}(Q S)=\frac{1}{\sqrt{2}} ; & E_{Q M}(R S)=\frac{1}{\sqrt{2}} \\
E_{Q M}(R T)=\frac{1}{\sqrt{2}} ; & E_{Q M}(Q T)=-\frac{1}{\sqrt{2}} .
\end{array}
$$

Summing it all up, we get

$$
E_{Q M}(Q S)+E_{Q M}(R S)+E_{Q M}(R T)-E_{Q M}(Q T)=2 \sqrt{2} .
$$

This is a very interesting result. Equation (4.10) is larger than the limit in inequality (4.7) [33, pp. 114-117] and it would appear that quantum mechanics is in contradiction with the "common-sense" rules we previously defined. How is this possible? Surely every step of our previous thought experiment was correct, right? We have to scrutinize our intuition of "common sense" and explicitly write down what we mean by it.

We will find two basic and intuitive ideas [33, p. 117]. So basic and intuitive, in fact, that EPR rather rejected quantum mechanics than forego them. The two ideas, stated informally, are:

1. Physical properties corresponding to the values $Q, R, S$, and $T$ exist no matter if we are observing them or not. This is called realism.

2. Alice's measurement does not influence the results of Bob's measurements and vice versa. This is, as we previously saw when discussing EPR, called locality.

The next section will give a formal definition of realism and locality and look closely at Bell's theorem. Then we will discuss the consequences of the theorem and put it to use in QKD. 


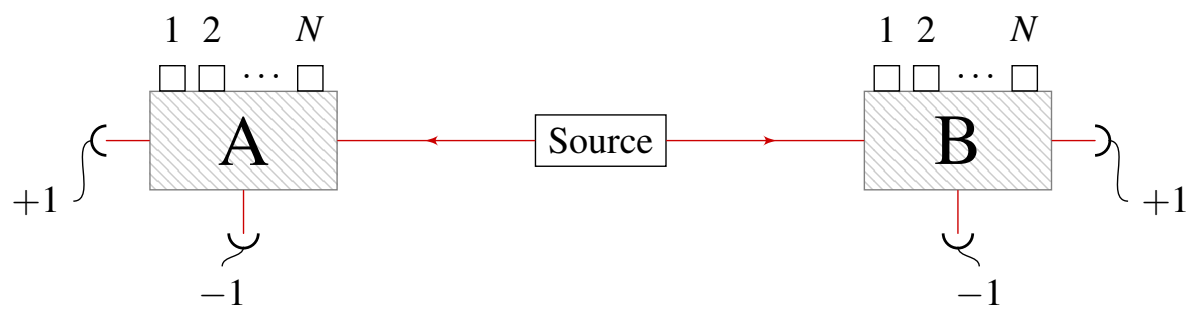

Figure 4.3: The black box method ignores the inner workings of the analysis stations and instead model them as boxes with $N$ push buttons and the outputs +1 and -1 .

\subsection{Bell's Theorem}

To give a definition of realism and locality, we first begin with a simplification of the way we model the interferometer and analysis stations. We will ignore everything about how the analysis stations are designed, except for its detectors and setting inputs. We instead model them as black boxes as shown in figure 4.3, where the interface consists of the quantum channel, a number of push buttons as input, and an +1 and -1 as output. This black box model simplifies the security analysis and will be useful for our discussion of Bell's theorem. We will also use the shorthand from equation (3.1) to make the equations more manageable.

We are now ready to give the formal definitions.

Definition 1 (Realism) A system is said to be realist if the analysis stations can be described by two families of random variables. A is Alice's analysis station with local setting $\phi_{A}$, and B is Bob's analysis station with local setting $\phi_{B}$. Both functions can depend on the hidden variable $\lambda$.

$$
A\left(\phi_{A}, \phi_{B}, \lambda\right) \text { and } B\left(\phi_{A}, \phi_{B}, \lambda\right)
$$

where the absolute values of the outcomes are bounded by 1 . The dependence on the hidden variable $\lambda$ is usually suppressed in the notation

Definition 2 (Locality) A system is said to be local if outcomes only depend on the local settings:

$$
A\left(\phi_{A}, \phi_{B}\right)=A\left(\phi_{A}\right) \text { and } B\left(\phi_{A}, \phi_{B}\right)=B\left(\phi_{B}\right) .
$$

A system that rules under locality and realism is said to be local realist. Looking back at hidden variables, we see that they (i) are defined as underlying mechanisms that result in a specific outcome, thereby implying realism, 
and (ii) only affect the local system, thereby implying locality. This shows that any system described by hidden variables is local realist.

We now move to the main result of this chapter and present a generalized version of Bell's theorem [35]. The generalization has been done in two steps, first by using an equivalent form introduced by Clauser, Horne, Shimony, Holt (CHSH) [43], and secondly by allowing more settings in the analysis stations as described by Pearle, Braunstein and Caves [44, 45]. The CHSH variant is used due to its flexibility in regards to experimental losses, and increasing the number of terms will help in our analysis of the Franson interferometer in section 5.1.

We begin by defining the Bell value:

Definition 3 (Bell value) The Bell value with $N \geq 2$ settings is defined as

$$
\begin{aligned}
S(N):=\mid & E\left(A_{1} B_{2}\right)+E\left(A_{3} B_{2}\right)|+| E\left(A_{3} B_{4}\right)+E\left(A_{5} B_{4}\right) \mid \\
& +\cdots+\left|E\left(A_{2 N-1} B_{2 N}\right)-E\left(A_{1} B_{2 N}\right)\right| .
\end{aligned}
$$

We now present the main theorem [35, 43-45]. be stated

Theorem 3 (Bell) The Bell value with $N$ settings for a local realist system with outcomes bounded in magnitude by 1 obeys

$$
S(N) \leq 2 N-2 .
$$

Remark 2 (CHSH) If only two settings are used in theorem 3 we get the original CHSH inequality [43]:

$$
\left|E\left(A_{1} B_{2}\right)+E\left(A_{3} B_{2}\right)\right|+\left|E\left(A_{1} B_{4}\right)-E\left(A_{3} B_{4}\right)\right| \leq 2 .
$$

All systems that are local realist are bound by theorem 3. What does it say about quantum mechanics? Well, if we choose measurement settings $\phi$ so that they are $\frac{\pi}{2 N}$ apart in the plane we have the following result from publication A:

Remark 3 Quantum mechanics predicts an N-term Bell value of

$$
S(N)=2 N \cos \left(\frac{\pi}{2 N}\right) .
$$

Since equation (4.14) violates inequality (4.12) we conclude that quantum mechanics violates local realism. Therefore, the hidden-variable description is incompatible with quantum mechanics and we must conclude that the 
EPR paradox is resolved by acknowledging that there does exist a special nonlocal connection in the Bell state. Quantum mechanics is not incomplete as suggested by EPR, but instead we see that nature cannot be fully described by realism and locality. Instead, there exists a phenomenon where distant particles form a system that cannot be divided into independent subsystems, and we call it entanglement.

The Bell state in equation (4.1) cannot be factored into smaller subsystems with the tensor product, and we will use this as a definition of entanglement. In contrast, a system that can be factorized is called separable. For instance, two qubits without entanglement have the following state vector:

$$
\begin{aligned}
& \frac{1}{2}(|00\rangle+|01\rangle+|10\rangle+|11\rangle) \\
& \quad=\frac{1}{\sqrt{2}}(|0\rangle+|1\rangle) \otimes \frac{1}{\sqrt{2}}(|0\rangle+|1\rangle) .
\end{aligned}
$$

Note that we were able to write equation (4.15) as a tensor product, which shows that two independent particles constitute a separable state.

Entanglement is indeed a peculiar phenomenon which has no equivalent in the classical world. The human mind is used to phenomena that are local and realist, which entanglement clearly is not. Nielsen and Chuang writes that entanglement can be used to create other peculiar phenomena, such as quantum teleportation and quantum error-correcting codes [33, pp. 25-28], all building blocks for a future quantum computer. We end this section with the following broad outlook:

Entanglement is a uniquely quantum mechanical resource that plays a key role in many of the most interesting applications of quantum computation and quantum information; entanglement is iron to the classical world's bronze age.

(Nielsen and Chuang [33, p. 11], emphasis in the original)

\subsection{A QKD Protocol Based on Bell's Theorem}

In 1991, Ekert [20] published a paper that detailed a QKD protocol that uses entanglement. As this discovery was made seven years after BB84, it was not the first QKD protocol, however it was the first that used Bell's theorem. In E91, there is no hierarchy between Alice and Bob. Instead, their roles are very similar and the state preparation task is instead given to a source device. We will in this section describe all steps of the E91 protocol. 


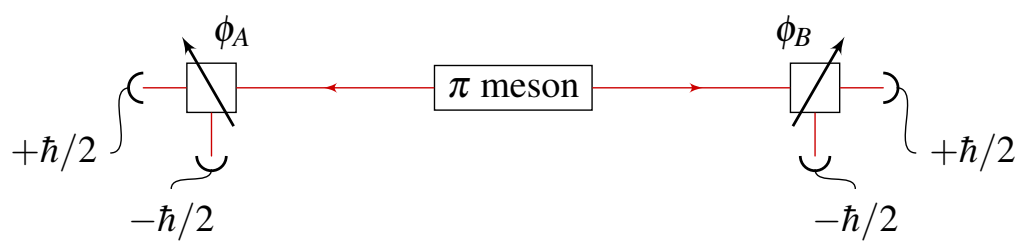

Figure 4.4: The E91 setup. A $\pi$ meson decays into two entangled particles that are measured along different axes by Alice and Bob. The resulting correlation violates Bell's inequality.

E91 works in a similar way to EPR thought experiment in section 4.1 and uses the same spin- $1 / 2$ particles ${ }^{\dagger}$. We therefore use a $\pi$ meson as an entanglement source just like EPR, and the E91 setup is depicted in figure 4.4.

An important difference to prepare-and-measure protocols is that no information is sent on the quantum channel [20]. Eve therefore has no motivation to eavesdrop; there is actually no information to eavesdrop upon. Instead, she can attempt other attacks such as attempting to replace the source with a Trojan device of her own.

Alice orients her detector to measure along one of the angles $\phi_{A_{1}}=0$, $\phi_{A_{2}}=\frac{1}{4} \pi$, and $\phi_{A_{3}}=\frac{1}{2} \pi$, while Bob chooses between $\phi_{B_{1}}=\frac{1}{4} \pi, \phi_{B_{2}}=\frac{1}{2} \pi$, and $\phi_{B_{3}}=\frac{3}{4} \pi$. Again, spin measurement gives one of the outcomes $+\hbar / 2$ or $-\hbar / 2$, but we will scale them to become \pm 1 . The quantity

$$
\begin{aligned}
E\left(A_{i} B_{j}\right)= & P\left(A_{i}=+1, B_{j}=+1\right)-P\left(A_{i}=-1, B_{j}=+1\right)- \\
& P\left(A_{i}=+1, B_{j}=-1\right)+P\left(A_{i}=-1, B_{j}=-1\right)
\end{aligned}
$$

is the expected value of the correlation between Alice and Bob when they use settings $i, j$. Using quantum rules we rewrite [20] equation (4.16) as

$$
E\left(A_{i} B_{j}\right)=-\cos \left(\phi_{A}-\phi_{B}\right) .
$$

After a number of measurements have been performed, Alice and Bob communicate their measurement settings over a public channel just like in BB84. Whenever Alice's and Bob's settings were equal (this happens in the cases $i=2, j=1$ and $i=3, j=2$ ), quantum mechanics predicts $E\left(A_{i} B_{j}\right)=-1$, i.e. perfect anticorrelation of their outcomes. These bits are

\footnotetext{
†It should be noted that while Ekert's original paper called for spin- $1 / 2$ particles, experimental realizations have instead used polarized photons as they are easier to handle.
} 
sorted out to later be distilled into a secret key, but in contrast to BB84 the rest of the bits are not discarded. Instead, they are used to check the Bell inequality. For these check bits, Alice and Bob publicly communicate their measurement outcomes, and quantum mechanics predicts the correlation $E\left(A_{i} B_{j}\right)=1 / \sqrt{2}$. Alice and Bob now compute

$$
S(2)=\left|E\left(A_{1} B_{1}\right)+E\left(A_{1} B_{3}\right)\right|+\left|E\left(A_{3} B_{1}\right)-E\left(A_{3} B_{3}\right)\right|=2 \sqrt{2} .
$$

This is the Bell value for the E91 protocol, and Alice and Bob now perform the Bell test. If $S(2)$ fulfills Bell's inequality (that is, no violation of equation (4.13)), Eve might have attempted an attack, and Alice and Bob have to stop communicating. This is the failure state of the test. A Bell value equal to $2 \sqrt{2}$, however, is a pass and they can continue with the rest of the protocol. In other words, the Bell test is a security test which must be passed before trusting the key output. The check bits can now be discarded and Alice and Bob continue with information reconciliation and privacy amplification to distill their shared secret key.

\subsection{Device-Independent QKD}

The black box model briefly introduced in section 4.3 allows the designer of a QKD system to greatly simplify the security analysis. It is a problem in both prepare-and-measure and entanglement-based protocols that some kind of trust must be placed in the source and the analysis stations. What if the analysis station manufacturer is infiltrated by Eve? Who do we trust?

In our discussion of the BB84 protocol in section 3.1, Alice and Bob perform measurements on a random subset of the raw bits and compare these with each other. They will then know [46] whether or not the communication is to be trusted. A full security proof of unconditional security in this scenario, however, requires intimate knowledge of the analysis stations and trust in their manufacturing process. In theory, there do exist proofs for QKD being unconditionally secure [47, 48], however the proofs assume ideal situations that cannot be achieved in the general experimental case. Scarani [49] describes a number of such complications.

The E91 protocol instead uses a violation of the Bell inequality to certify the system as secure. Instead of having to perform tedious proofs that involve the complicated inner workings of the analysis stations like in BB84, the Bell test only involves measurement outcomes. After Ekert's initial publication [20], subsequent works by various authors provided a few more 
pieces to the puzzle [50,51] but as Acín et al. [52] points out, these results either did not give the whole picture or prove the general case with noise. Instead, a revolution came with the development of Device-Independent Quantum Key Distribution (DI-QKD), a term coined by Acín et al. [52]. The history of events that led up to this new idea is documented by Scarani [49, pp. 56-58].

DI-QKD takes a step back from traditional QKD and only assumes Eve to be constrained by quantum mechanics. This is a considerable relaxation over traditional QKD protocols that not only assumes hostile control of the source, but also of the quantum channel and the and analysis stations. To only be constrained by quantum mechanics means Eve can do almost anything she wants with only a few exceptions such as "no signaling faster than the speed of light". Acín et al. [52] write:

The only data available to Alice and Bob to bound Eve's knowledge are the observed relation between the measurement settings and outcomes, without any assumption on how the measurements are actually carried out or on what system they operate.

In addition to the above security assumption of Eve obeying the laws of quantum mechanics, Alice and Bob are also assumed to be free to choose secret measurement settings and that the outcomes they measure are kept secret [52]. Usually, these two last assumptions are referred to as saying that no information should leak out of Alice's and Bob's laboratory.

It is important to note that traditional QKD protocols fail to provide security under device-independent security assumptions. To illustrate this, we will analyze an entanglement-based variant of the BB84 protocol and follow an example adapted from Pironio et al. [46]. This modified BB84 protocol uses $N=2$ measurement settings and produces the outcomes +1 and -1 . For simplicity, we assume ideal experimental conditions so that no outcome has the value 0 .

Alice and Bob perform measurements on polarized photons, and the setting $\phi_{1}$ means "measure polarization along the $x$ axis" while $\phi_{2}$ means "measure polarization along the $z$ axis". Suppose now that they observe perfectly correlated outcomes if Alice's setting $i$ equals Bob's setting $j$ and uncorrelated random outcomes if $i \neq j$. Rewriting this in Dirac's bra-ket notation we get

$$
\begin{gathered}
\left\langle\psi\left|\phi_{1} \otimes \phi_{1}\right| \psi\right\rangle=\left\langle\psi\left|\phi_{2} \otimes \phi_{2}\right| \psi\right\rangle=1 \\
\left\langle\psi\left|\phi_{1} \otimes \phi_{2}\right| \psi\right\rangle=\left\langle\psi\left|\phi_{2} \otimes \phi_{1}\right| \psi\right\rangle=0,
\end{gathered}
$$


and the only quantum state fulfilling these conditions is the Bell state [46] from equation (4.1). With this knowledge, Alice and Bob can be sure that the system is entangled and that Eve has played no hidden-variable trickery with the source device.

If the same system is used with the security assumptions of DI-QKD Alice and Bob will have a problem. They can no longer assume that $\phi_{1}$ and $\phi_{2}$ corresponds to the previously defined measurement operations, nor can they even know what is actually being measured upon. With the conditions favoring her this time, Eve makes the source source create a state consisting of four qubits as described by Acín, Gisin, and Masanes [53]. She then sends two qubits to Alice and two to Bob, and when they perform their measurements they will get the same correlations as before. Again, using equation (4.19) they are lead to believe that the quantum state of the system is the Bell state. However, this is not the case as the quantum state prepared by Eve is separable and tailored to leak information from Alice's and Bob's analysis stations. The key generated by Alice and Bob will therefore be known to Eve [53]! This example shows that relaxing the requirements on the measurement devices leads to traditional QKD protocols becoming insecure. Specifically, Eve was able to fool Alice and Bob using a higherdimensional system than they expected, thereby exposing the secret key bits.

If a suitable protocol is used, DI-QKD works because any state producing the Bell value $2 \sqrt{2}$ is the Bell state [54] (or a state equivalent to it). Non-locality is "the physical principle on which all device-independent security proofs are based" [46]. Indeed, the security assumptions of DI-QKD only work for protocols that violate Bell's inequality and as we previously saw, BB84 is not such a protocol.

An important fact about Bell's theorem is that it is independent of quantum mechanics. The derivation in section 4.2 only assumed locality and realism so Bell inequality applies to any "common-sense" system with remote correlations. The only time we involve quantum mechanics in relation to Bell's theorem is when we show that quantum mechanics is in violation of $i t$. Analyzing the Bell value does not involve thinking about "paths in an interferometer", "Hilbert spaces", "photons" and so on. Therefore, if the measurement outcomes of a QKD system violates the Bell inequality, it follows that the state it is not classical and can be certified as secure (bar any loopholes discussed in section 4.6).

We can conclude that DI-QKD allows Alice and Bob to use Bell's inequality as a security test for a whole QKD system without requiring trust 
in either the source, quantum channels or the analysis stations. As long as they are free to secretly choose settings for their analysis station and observe a violation of Bell's inequality, they can rule out the presence of Eve.

\subsection{Loopholes in Bell Experiments}

But power supplies make noise, and not the same noise for the different voltages needed for different polarizations. So, we could literally hear the photons as they flew, and zeroes and ones made different noises. Thus, our prototype was unconditionally secure against any eavesdropper who happened to be deaf!

(Gilles Brassard [34, p. 20], emphasis in the original)

The theory of DI-QKD is appealing due to its elegance and simplicity. In theory, the Bell test works because no hidden variables can reproduce Alice's and Bob's measurement outcomes. In practice, however, there will be complications introduced by the reality of performing delicate physical experiments in a real laboratory. The above story told by Gilles Brassard highlights such a reality, where the sound made by the power supplies controlling the polarizers leaked information about the setting. This is a telling example of a loophole, an unexpected situation that might not be apparent in theory. Loopholes come in many shapes and forms, and this section will discuss some that are relevant to our goals.

In 1970, Pearle realized that an apparent violation of Bell's inequality can be fabricated from a local realist system. This is done by carefully excluding some of the events when computing the Bell value [44]. Pearle's example shows that care must be taken whenever implementing a Bell test as losing too many events causes a "fake" violation. This loophole, the detection loophole, is an inherent problem in any experimental test of Bell's inequality as optical fibers, beam splitters, and detectors have inherent losses. We must therefore find what losses are acceptable without the Bell inequality becoming unusable.

Generally speaking, a loophole causes the Bell test to give a false positive; incorrectly certifying a system as not being local and realist. Let us use the results from publication A and Cabello, Larsson, and Rodríguez [55] to show the effects of the detection loophole on the Bell inequality. In fact, theorem 3 is changed to the following:

Theorem 4 A local realist model with inefficiency has the following three properties: 
Realism. Outcomes are given by random variables on subsets of detection (in a probability space $(\Lambda, \mathscr{F}, P)$ ),

$$
A\left(\phi_{A}, \phi_{B}, \lambda\right) \text { on } \Lambda_{A, \phi_{A}, \phi_{B}} \quad \text { and } \quad B\left(\phi_{A}, \phi_{B}, \lambda\right) \text { on } \Lambda_{B, \phi_{A}, \phi_{B}} .
$$

Locality. Outcomes and detections do not depend on the remote settings,

$$
\begin{array}{lll}
A\left(\phi_{A}, \phi_{B}\right)=A\left(\phi_{A}\right) & \text { on } & \Lambda_{A, \phi_{A}, \phi_{B}}=\Lambda_{A, \phi_{A}} \\
B\left(\phi_{A}, \phi_{B}\right)=B\left(\phi_{B}\right) & \text { on } & \Lambda_{B, \phi_{A}, \phi_{B}}=\Lambda_{B, \phi_{B}} .
\end{array}
$$

Efficiency. There is a lower bound to the detection efficiency,

$$
\eta=\min _{\substack{\text { settings } \\ \text { local sites }}} P(\text { coincidence } \mid \text { local detection }) .
$$

The outcomes from a local realist model with inefficiency obey

$$
\begin{aligned}
\mid E & \left(A_{1} B_{2} \mid \text { coinc. for } A_{1} \text { and } B_{2}\right) \\
& +E\left(A_{3} B_{2} \mid \text { coinc. for } A_{3} \text { and } B_{2}\right) \mid \\
& +\mid E\left(A_{3} B_{4} \mid \text { coinc. for } A_{3} \text { and } B_{4}\right) \\
& +E\left(A_{5} B_{4} \mid \text { coinc. for } A_{5} \text { and } B_{4}\right) \mid+\cdots \\
& +\mid E\left(A_{2 N-1} B_{2 N} \mid \text { coinc. for } A_{2 N-1} \text { and } B_{2 N}\right) \\
& -E\left(A_{1} B_{2 N} \mid \text { coinc. for } A_{1} \text { and } B_{2 N}\right) \mid \\
& \leq 2-2 N+\frac{4}{\eta}(N-1)
\end{aligned}
$$

We see that theorem 3 is the special case of the more general theorem 4 with $\eta=1$. As the detection efficiency $\eta$ decreases to zero, the right hand side of inequality (4.20) goes to infinity, and the power of the inequality is eliminated. As we saw in remark 3, quantum mechanics predicts the Bell value $S(N)^{Q M}=2 N \cos \frac{\pi}{2 N}$ and we can therefore find a critical $0<\eta<1$ where the quantum prediction $S(N)^{Q M}$ no longer violates local realism. Indeed [55], this value is

$$
\eta_{c r i t}=\frac{2}{\frac{N}{N-1} \cos \left(\frac{\pi}{2 N}\right)+1},
$$

and thus for any $0 \leq \eta \leq \eta_{\text {crit }}$ the Bell test will be of no use for security testing. Any experimental implementation of the Bell test wanting to eliminate 
the detection loophole must therefore have an average detection efficiency well above $\eta_{\text {crit }}$. When using the original CHSH inequality from remark 2, the critical value is as high as $82.83 \%$ and achieving that efficiency in experiment is a challenge even today.

With this simple example we see that loopholes can cause problems for our goal of creating secure quantum key distribution. Inefficient detection is, however, not the only way to get around the Bell test; there are many more loopholes that need to be closed before we have unconditional security.

The first experimental evidence for quantum mechanics violating Bell's theorem is due to Aspect, Grangier, and Roger [56] and Aspect, Dalibard, and Roger [57] in the early 1980:s. These early experiments did show a violation, but the detection efficiency is too low to give conclusive evidence. Still, the Aspect experiments were pioneering efforts that had enormous impact on the field [58, p. 11]. The experiments did, however, close the locality loophole that can arise if a local setting at an analysis station has time to travel to the remote station before measurement occurs. In that case there is nothing that rules out that a particle "learns" the setting and knowingly chooses to give a certain outcome.

The detection and locality loopholes are only two examples of ways in which Nature could play tricks on an experimenter. Other examples not discussed here include the superdeterminism [59] and fair sampling loopholes. While almost all loopholes can be handled [58, p. 28] there has been no experiment closing all of them simultaneously. Therefore, there does not yet exist irrefutable proof that Bell's inequality is violated by quantum mechanics in experiment. A lot of work on closing all loopholes is ongoing, though, and we will end our discussion on loopholes with a quote by Leggett [60, pp. 1469-1470]:

Over the past thirty years a very large number of experiments have been conducted with the aim of testing the predictions of quantum mechanics against those of local hidden-variable theories, and while to the best of my knowledge no single existing experiment has simultaneously blocked all of the so-called "loopholes" (detector efficiency, random choice of setting, etc.), each one of those loopholes has been blocked in at least one experiment $[\ldots]$. Thus, to maintain a local hidden-variable theory in the face of the existing experiments would appear to require belief in a very peculiar conspiracy of nature. 


\section{Chapter 5}

\section{Energy-Time Entanglement}

Up to this point we have discussed entanglement by means of polarization as seen in the BB84 protocol of section 3.1. Even though this method is a viable building block for $\mathrm{QKD}$, we need protocols that are robust not only in a laboratory, but over long distances and in tough conditions as well. Polarization-based protocols have drawbacks due to the fact that the degree of polarization through an optical fiber is heavily dependent on environmental factors [61]. Changes in temperature [62], magnetic field [63], and mechanical stress [64] might influence the relative polarization as photons travel through the fiber.

In an uncontrolled environment, these changes must be quantified and compensated for. Otherwise, the measurement outcomes for Alice and Bob will drift in an uncontrolled manner and give rise to errors in the bit output. The standard way of correcting polarization drift is through a mechanical stretcher, where an optical fiber is stretched by a piezo-electric device [61]. Unfortunately, this compensator is a device that requires moving parts and would be complicated and expensive to manufacture and maintain in a largescale deployment.

In recent years there has been an increase in interest for replacing polarization with a method not requiring expensive compensating equipment. This thesis will discuss one such proposal where the conjugate variables of energy and time are used instead of non-orthogonal polarization states. This method can be used with entanglement and is therefore called energy-time entanglement. Originally, the idea was introduced unintentionally by Einstein in the clock-in-a-box thought experiment as early as 1930 [41, pp. 94-97, 65]. The thought experiment was intended to find a contradiction in quantum mechanics, but Bohr was famously able to resolve Einstein's riddle by 


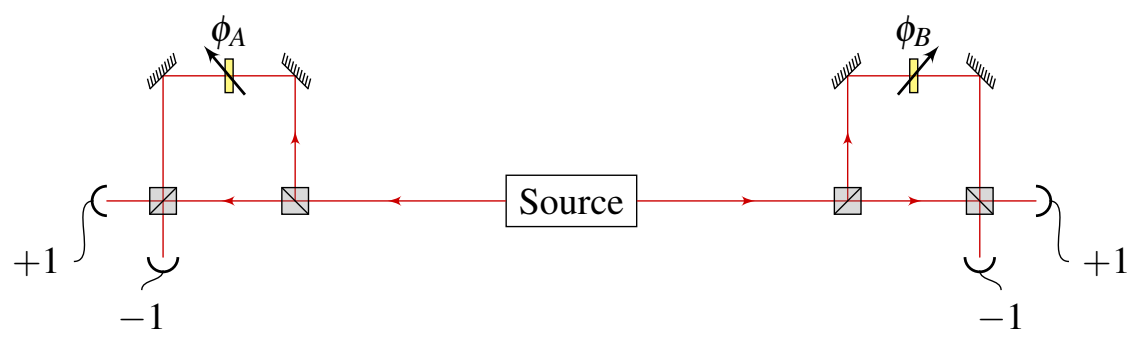

Figure 5.1: The Franson interferometer. The source emits time-correlated photons that are sent to Alice and Bob. At their respective analysis stations, they perform measurements with settings $\phi_{A}$ and $\phi_{B}$ and record the outcome as well as time of detection.

showing that energy and time are conjugate variables just like position and momentum.

\subsection{The Franson Interferometer}

The first proposal to build a device based on energy-time entanglement was published in 1989 by Franson [23]. This device, depicted in figure 5.1, consists of the usual parts found in an entanglement-based qkd setup; a souce and two analysis stations. Franson's original proposal described the source as follows: An excited atom is in a relatively stable high-energy state at time $t=0$ with a relatively long average lifetime $\Delta T$. A photon is emitted, which brings the atom to a middle level with a short lifetime $\tau \ll \Delta T$. When the atom goes back to the ground state a second photon is emitted. The atom is therefore a three-level system with two photons emitted within a small time window $\tau$. The source will therefore emit two photons almost simultaneously, but the time of emission is uncertain over a long timescale $\Delta T$.

Alice and Bob each have an identical analysis stations consisting of two optical paths of different length. The upper path is longer than the lower by a path difference $c \Delta T$ and also contains a variable phase modulator (setting) $\phi$. The beam is split into the two paths by a 50:50 beam splitter and they are intermixed using another one. The second beam splitter does not combine the beams into one, but has two outputs. Each of these beam splitter outputs is connected to one of the two photodetectors +1 or -1 .

This whole device is called the Franson interferometer and now that the 
physical layout has been established, Alice and Bob use it in the following way: The three-level atom in the source is excited to the semi-stable highenergy state described above. An unknown moment in time later (expected value $\Delta T$ ), the two photons are emitted and are sent to Alice and Bob, who randomly choose the settings $\phi$ for their analysis stations.

We will now study the effect of the analysis station on the entangled state. The path difference results in a possible delay to the photon, and the phase modulator setting $\phi$ affects the way the paths combine at the second beam splitter. Remember that photons are quantum objects and do not take a specific "path" through the analysis station. At the end of the analysis station the photon is detected by one of the two detectors, resulting in a measurement outcome of +1 or -1 . When registering these detections, Alice and Bob also record the time the detection occurred.

The photons created at the source have now been measured, and one round of the Franson interferometer is therefore complete. This process is now repeated a number of times, so that Alice and Bob each have a list of measurement outcomes and detection times. The outcomes +1 and -1 constitutes the raw key at this point, and they now begin the sifting process. Before this can be done, however, they must first perform a postselection step in which events arriving at differing times at Alice's and Bob's detectors are discarded. This stands in contrast to the BB84 and E91 protocols where sifting can be done immediately. The postselection will have serious consequences to the security of Franson-based QKD as we will discuss in sections 5.2 and 5.3 .

If the detection times are equal there is quantum-mechanical interference between the two cases (i) early source emission, photons were delayed by both Alice's and Bob's analysis stations and (ii) late source emission, neither analysis station caused a delay. Alice and Bob will not know which of the two cases that actually occurred, but if there is a detection time mismatch, however, calculating the emission time is trivial and there will be no interference. Alice and Bob must therefore remove all events with differing detection times in order to force interference and this is the actual postselection.

Now Alice and Bob compute the correlation between the postselected (coincident) measurement outcomes:

$$
E\left(A\left(\phi_{A}\right) B\left(\phi_{B}\right) \mid \text { coincident detection time }\right)=\cos \left(\phi_{A}+\phi_{B}\right) .
$$

The complete derivation can be found in Franson's paper [23]. Equation (5.1) shows that, if Alice's and Bob's setting are oppsosite $\left(\phi_{A}+\phi_{B}=0\right)$, the 
measurement outcomes are perfectly correlated. Similarily, for settings $\phi_{A}+\phi_{B}=(2 n+1) \pi$ where $n$ is an integer, Alice's outcomes will be opposite to Bob's.

Alice and Bob now perform a Bell test in a similar way to the E91 protocol discussed in section 4.4. After publicly announcing the analysis station settings $\phi_{A}$ and $\phi_{B}$, Alice and Bob use the measurement outcomes with $\phi_{A} \neq \phi_{B}+n \pi$ as check bits, and using equation (5.1) the quantummechanical prediction of the two-term* Bell value $S(2)$ is $2 \sqrt{2}$. According to Franson [23, p. 2207],

[...] the quantum-mechanical predictions violate Bell's inequality and are inconsistent with any local hidden-variable theory.

If Alice and Bob measure an experimental Bell value that does not violate the CHSH ineqality it is possible that Eve is attacking the system and Alice and Bob will have to abort the communication. If, however, the test passes, they can continue extracting the secret key. By performing a bit flip on the anticorrelated bits, Alice and Bob can continue with information reconciliation and privacy amplification to generate a secret key.

In other words, the Franson interferometer relies on the Bell test for security. Again, it is impossible to distinguish between an eavesdropper and experimental noise and both will have the effect of lowering the measured Bell value. If it no longer violates the CHSH inequality, they must abort the transmission.

We have now seen that we can test Bell's inequality using energy and time instead of polarization. Using the Franson interferometer we can also perform QKD to generate a secret key and this has also been tested experimentally QKD [24-31]. Keep in mind, however, that the Franson setup uses a postselection step. On average, this results in $50 \%$ of the events being discarded and the consequences are severe, as we will see in the next section.

\subsection{Loopholes in the Franson Interferometer}

We have now seen that the Franson interferometer is believed (i) to violate local realism, and (ii) to allow secure energy-time-entangled QKD. However,

*While Franson does not explicitly state that the Bell value should be computed with two settings $(N=2)$ it is clear from context that this is what is intended. 
as we discussed in section 4.6, the Bell test must always be viewed in the light of loopholes, and the detection loophole has particular consequences for the Franson setup as is shown in publication A [66].

We saw in theorem 4 that the the lower bound on detection efficiency is $82.83 \%$ when using the original $\mathrm{CHSH}$ inequality. The Franson interferometer, with its $50 \%$ postselection step, falls well below this limit and in publication A we thoroughly discuss the consequences of this postselection. In essence it allows an attacker to "fake" the Bell violation and thereby break the security of the system.

This weakness is called the postselection loophole and is related to the detection loophole. With postselection taken into account, the $\mathrm{CHSH}$ inequality for the Franson interferometer is

$$
\begin{aligned}
& \mid E\left(A_{1} B_{2} \mid \text { coinc. for } A_{1} \text { and } B_{2}\right) \\
& \quad+E\left(A_{3} B_{2} \mid \text { coinc. for } A_{3} \text { and } B_{2}\right) \mid \\
& \quad+\mid E\left(A_{1} B_{4} \mid \text { coinc. for } A_{1} \text { and } B_{4}\right) \\
& \quad+E\left(A_{3} B_{4} \mid \text { coinc. for } A_{3} \text { and } B_{4}\right) \mid \leq 6 .
\end{aligned}
$$

The algebraic maximum for the left-hand side of inequality (5.2) is 4 , so the inequality reduces to just the trivial " $\leq 4$ ". Therefore, no state, whether quantum or classical, can violate local realism in the Franson interferometer.

If we, however, let the analysis stations change settings very quickly so that a new setting is chosen randomly as the photon is travelling from the source, publication A shows that the situation is somewhat better. The corresponding Bell inequality becomes

$$
\begin{aligned}
& \mid E\left(A_{1} B_{2} \mid \text { coinc. for } A_{1} \text { and } B_{2}\right) \\
& \quad+E\left(A_{3} B_{2} \mid \text { coinc. for } A_{3} \text { and } B_{2}\right) \mid \\
& \quad+\mid E\left(A_{1} B_{4} \mid \text { coinc. for } A_{1} \text { and } B_{4}\right) \\
& \quad+E\left(A_{3} B_{4} \mid \text { coinc. for } A_{3} \text { and } B_{4}\right) \mid \leq 3 .
\end{aligned}
$$

Still, the quantum prediction $2 \sqrt{2}$ falls short of this bound and gives violation of local realism. The original $\mathrm{CHSH}$ inequality therefore appears to be inadequate for the Franson interferometer. In fact, publication A presents a Local Hidden Variable (LHV) model whose predictions perfectly match the quantum-mechanical predictions.

In publication A we also show that it is possible, in theory, to re-establish security by discarding the device-independent security assumptions and instead require the path inside the analysis station to be a realist property. This 
"path realism" approach allows the right-hand side of the CHSH inequality to be lowered back to 2 which lets quantum mechanics violate local realism. The problem, of course, is that we lose the benefits of device-independence and again have to consider every component in a security proof. In this situation it is also hard to prove that the path taken by a photon really is independent of the setting.

It appears clear that the original $\mathrm{CHSH}$ inequality is inadequate as a security test in the Franson interferometer. Publication A therefore presents several alternative setups that would re-establish unconditional security. If the Bell test is replaced with a test with more settings, i.e. theorem 3 with $N \geq 3$, we show that quantum mechanics again violates local realism. However, we also show that this approach requires almost ideal experimental conditions as losses must be almost negligible. An alternative solution would be to replace the Franson interferometer with a device not requiring postselection. So-called genuine energy-time entanglement $[67,68]$ is one such proposal.

\subsection{Realization of Loopholes}

While publication A shows that there exists a LHV model giving the same predictions as the Franson interferometer, publication B [69] explicitly details how such a model can be implemented. The goal of this paper is to make it clear that the Franson interferometer together with the CHSH inequality can be attacked by a third party even though an apparent Bell violation occurs.

The LHV model dictates which outcomes should occur when, and our attack in publication B uses time-delayed pulses of classical lights. These pulses are sent by our Trojan source device, and in order to produce the correct pulses one needs a good understanding of how the analysis stations work. The most important part of any interferometer is a beam splitter, sometimes called a "half-silvered mirror", which splits and combines light beams. In the Franson setup, each analysis station contains two beam splitters so the entire interferometer uses four.

Beam splitters can be manufactured in a variety of ways, but the operation is the same. Two edges are inputs and two are outputs, and along the diagonal line there is a mirror-like interface. Each input beam is split into the two output beams, however the output intensity also depends on interference which in turn depends on the relative phases of the incident beams. The 


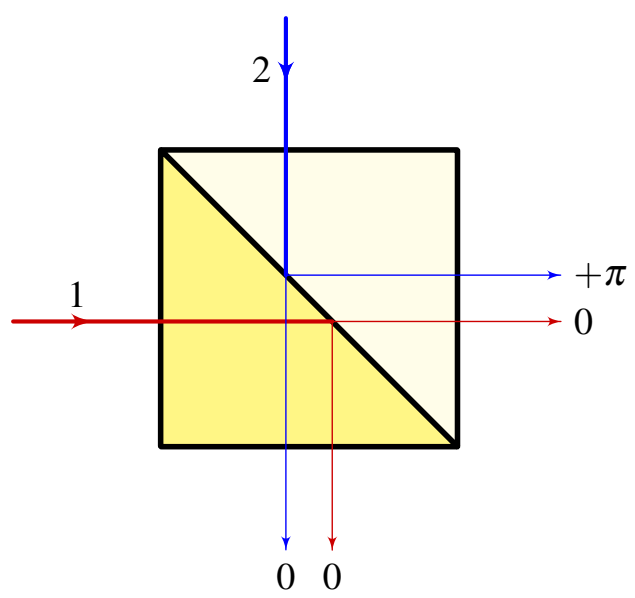

Figure 5.2: Schematic of a beam splitter manufactured by joining two triangular prisms with different refractive indices. The beam splitter depicted here has a high refractive index in the lower left region and a low refractive index in the top right. Two incident beams are combined into two output beams. The beam from the left receives no phase shift but the beam from the top receives a $\pi$ phase shift when reflected off the higher refractive index region.

easiest way to understand how beam splitters affect the phase is to consider a beam splitter consisting of two triangular pieces of glass with different indices of refraction as depicted in figure 5.2.

We let the first incident beam have intensity $I / 2$ and enter the region with higher refractive index from left. The second incident beam, also with intensity $I / 2$, enters from the top. Assuming we are dealing with an ideal $50: 50$ beam splitter, each beam is split into two at the interface without loss. According to Träger [70, pp. 124-125], the total intensity of all output beams remains $I$ so conservation of energy is fulfilled. For the first beam there is no reflection off a transition from low to high refractive index, no phase shift occurs. The second beam also gets no phase shift when transmitted into the high index region, however the component that is reflected has its phase shifted by $\pi$. Therefore, three of the output beams have no change in phase while the fourth has. The output intensities $I_{R}$ (right exit) and $I_{B}$ (bottom exit) then depend on the phases $\varphi_{1}$ and $\varphi_{2}$ of the input beams in the 
following way:

$$
\begin{aligned}
I_{R} & =\frac{I}{2}\left(1+\cos \left(\varphi_{1}-\varphi_{2}+\pi\right)\right) \\
I_{B} & =\frac{I}{2}\left(1+\cos \left(\varphi_{1}-\varphi_{2}\right)\right) .
\end{aligned}
$$

We see that the total output intensity $I_{R}+I_{B}$ sums to $I$ independently of the phases $\varphi_{1}$ and $\varphi_{2}$ which shows that our equation (5.4) conserves energy. In order to control the constructive and destructive interference at the output ports we therefore must produce beams of light with a relative phase difference of either 0 or $\phi / 2$. The first case, zero relative phase, will direct all incoming light out of the first port while a relative phase of $\phi / 2$ does the opposite.

We have now established the relative phases of the incoming light beams to the second beam splitter at the analysis station. Working backwards, we see that the two paths have different time delays and phase shifts. If a short pulse of light enters the first beam splitter, two pulses will enter the second beam splitter. If we instead send two pulses separated by $\Delta T$ into the analysis station, we will have control of the second beam splitter. The relative phase of the two input pulses will then affect the intensity at each detector as described by equation (5.4) and can be used as a hidden variable. There are some further details needed to fully implement this attack but they are all discussed in publication B.

Our attack on the Franson interferometer is not only performed in theory, but we have shown it experimentally as well. Using a fiber-optic setup we were able to not only violate Bell's inequality, but almost reach the algebraic maximum of $S(2)=4$ as well. Whenever a Bell experiment is performed, noise has the effect of lowering the measured Bell value and this reduces the effectiveness of Alice's and Bob's Bell test and, given enough noise, forces them to stop communicating. Since Eve is indistinguishable from noise, she wants to perform her attack without the corresponding reduction in the Bell value. But the high level of Bell violation allows her to tailor the attack so that Alice and Bob reach exactly $2 \sqrt{2}$ even when there is noise on the channel.

Just like in publication A we conclude publication B by suggesting stronger alternatives to the Franson setup. 


\section{Chapter 6}

\section{Conclusions and Future Work}

We have now seen a short history of cryptography leading up to the modern developments of energy-time entangled and device-independent quantum key distribution. In essence, QKD is the ultimate form of secret communication as it remains secure to any eavesdropper, even in a future where quantum computers are a reality. The slogan of QKD has long been "security based on the laws of physics" [49], but as pointed out by Scarani and Kurtsiefer [71], the same laws "do not prevent someone from reading the outcomes of a detector". Just because the theoretical security can be proved it does not mean the system is immune to real-life attacks such as the one in publication B.

In chapter 1 we introduced Schneier's Law which states that cryptographic systems must be exposed to cryptanalysis and tested extensively before using them for sensitive information. The law obviously applies to QKD as well, but as the field is relatively new there is a chance that wonderful discoveries will be made in the coming years. We cannot predict the ongoing rapid development and therefore must subject our QKD devices to extra scrutiny before marketing them to the public.

DI-QKD and energy-time entanglement are elegant concepts that could pave the way for a new generation of efficient and robust devices with very high security promises, but first we must analyze every detail and resolve any issues that might be found. The Franson interferometer, being the pioneering implementation of energy-time entanglement, must be credited with ushering in this new era in quantum information. However, as we have clearly seen in this thesis, there exist issues that could allow Eve to successfully attack a system without Alice and Bob even noticing.

The origins of our attack on the Franson interferometer in publications A and B can be traced back to 1999, when Aerts et al. [72] investigated LHV 
models and energy-time entanglement. Many years have passed since this publication, yet Franson experiments using the $\mathrm{CHSH}$ inequality continue to be performed where the violation is assumed to rule out local realism [24-31]. As we have seen in this thesis, however, the security Franson interferometer can be broken as shown theoretically in publication A and demonstrated experimentally in publication $\mathrm{B}$.

It could also be tempting to modify the interferometer setup to detect our specific attack. Such a modification would certainly make the specific method in publication B impossible, but it does not give the user any confidence in the future security of the system. An attacker could then modify his attack to circumvent the detection system and the result is a never-ending cat-and-mouse game. The security proof relies on a violation of the Bell inequality and can not be substituted by any patchwork of systems looking for specific attacks. Instead, we must continue to rely on primitives that can be proven secure and make them robust enough to work in experimental, non-ideal conditions.

The device-independent method is appealing due to its elegance and reliance on one single and provable theorem. In the future, this elegance should make it possible to give a thorough proof of unconditional security of the Franson interferometer with modified Bell tests.

There are a number of QKD protocols not discussed in this thesis. Future work could therefore also include investigating if it is possible to prove their unconditional security and give explicit noise limits, similar to the $11 \%$ upper limit of the BB84 protocol. Protocols to investigate include COW [21] and DPS [22]. For the COW protocol, some early results are available regarding necessary conditions for a full security proof [73]. 


\section{Bibliography}

[1] M. Willett. "Cryptography old and new". In: Computers \& Security 1.2 (June 1982), pp. 177-186. DOI: 10 . 1016/0167-4048(82) 90010-4.

[2] G. F. Strasser. "The rise of cryptology in the European Renaissance". In: The History of Information Security. Elsevier, 2007, pp. 277-325. DOI: $10.1016 / \mathrm{b} 978-044451608-4 / 50012-2$.

[3] T. Kelly. "The Myth of the Skytale". In: Cryptologia 22.3 (July 1998), pp. 244-260. DOI: 10.1080/0161-119891886902.

[4] D. Davies. "A brief history of cryptography". In: Information Security Technical Report 2.2 (Jan. 1997), pp. 14-17. DOI: 10.1016/s13634127 (97)81323-4.

[5] D. Kahn. The codebreakers: the story of secret writing. Macmillan, 1967. 1216 pp.

[6] F. Petitcolas, R. Anderson, and M. Kuhn. "Information hiding-a survey”. In: Proc. IEEE 87.7 (July 1999), pp. 1062-1078. DOI: 10.1109/ 5.771065 .

[7] S. Singh. The code book: the evolution of secrecy from Mary, Queen of Scots, to quantum cryptography. New York: Doubleday, 1999. ISBN: 0385495315.

[8] S. Nakamoto. "Bitcoin: A peer-to-peer electronic cash system". In: Consulted 1.2012 (2008), p. 28.

[9] W. Trappe and L. Washington. Introduction to Cryptography: With Coding Theory. Featured Titles for Cryptography Series. Pearson Prentice Hall, 2006. ISBN: 9780131862395.

[10] B. Schneier. Memo to the Amateur Cipher Designer. Oct. 15, 1998. URL: https : / /www . schneier . com/crypto-gram/archives / 1998/1015.html\#cipherdesign (accessed 01/16/2015). 
[11] K. de Leeuw. "Introduction". In: The History of Information Security. Elsevier, 2007, pp. 1-25. DOI: 10 . 1016/b978-044451608-4 / 50002-x.

[12] W. Diffie and M. Hellman. "New directions in cryptography". In: IEEE Transactions on Information Theory 22.6 (Nov. 1976), pp. 644654. DOI: $10.1109 /$ tit.1976.1055638.

[13] R. L. Rivest, A. Shamir, and L. Adleman. "A method for obtaining digital signatures and public-key cryptosystems". In: Commun. ACM 21.2 (Feb. 1978), pp. 120-126. DOI: 10.1145/359340.359342.

[14] B. Schneier. Applied cryptography: protocols, algorithms, and source code in C. Wiley, 1996. ISBN: 9780471128458.

[15] P. W. Shor. "Algorithms for Quantum Computation: Discrete Logarithms and Factoring". In: Proceedings of the 35th Annual Symposium on Foundations of Computer Science. Santa Fe, NM: IEEE Computer Society Press, Nov. 1994, pp. 124-134.

[16] E. Martín-López, A. Laing, T. Lawson, R. Alvarez, X.-Q. Zhou, and J. L. O’Brien. 'Experimental realization of Shor's quantum factoring algorithm using qubit recycling”. In: Nature Photonics 6.11 (Oct. 2012), pp. 773-776. DOI: 10.1038/nphoton.2012.259.

[17] D. J. Bernstein, J. Buchmann, and E. Dahmen. Post-quantum cryptography. Springer, 2009.

[18] R. J. McEliece. "A public-key cryptosystem based on algebraic coding theory”. In: DSN progress report 42.44 (1978), pp. 114-116.

[19] C. H. Bennett and G. Brassard. "Quantum cryptography: Public key distribution and coin tossing". In: Proc. of the IEEE Int. Conf. on Computers, Systems, and Signal Processing. 175-179. Bangalore, India: IEEE New York, 1984.

[20] A. K. Ekert. "Quantum cryptography based on Bell's theorem”. In: Phys. Rev. Lett. 67 (1991), pp. 661-663. DOI: 10.1103/PhysRevLett. 67.661 .

[21] D. Stucki, N. Brunner, N. Gisin, V. Scarani, and H. Zbinden. "Fast and simple one-way quantum key distribution". In: Applied Physics Letters 87.19 (2005), p. 194108.

[22] K. Inoue, E. Waks, and Y. Yamamoto. "Differential phase-shift quantum key distribution”. In: Photonics Asia 2002. International Society for Optics and Photonics. 2002, pp. 32-39. 
[23] J. D. Franson. "Bell inequality for position and time". In: Physical Review Letters 62.19 (1989), pp. 2205-2208. DOI: 10 . 1103/ PhysRevLett.62.2205.

[24] W. Tittel, J. Brendel, H. Zbinden, and N. Gisin. "Violation of Bell Inequalities by Photons More Than $10 \mathrm{~km}$ Apart". In: Phys. Rev. Lett. 81.17 (Oct. 1998), pp. 3563-3566. DOI: 10.1103/physrevlett. 81.3563.

[25] W. Tittel, J. Brendel, B. Gisin, T. Herzog, H. Zbinden, and N. Gisin. "Experimental demonstration of quantum correlations over more than 10 km”. In: Physical Review A 57.5 (May 1998), pp. 3229-3232. DOI: 10.1103/physreva.57.3229.

[26] W. Tittel, J. Brendel, H. Zbinden, and N. Gisin. "Quantum Cryptography Using Entangled Photons in Energy-Time Bell States". In: Phys. Rev. Lett. 84.20 (May 2000), pp. 4737-4740. DOI: 10.1103/ physrevlett.84.4737.

[27] Z. Ou, X. Zou, L. Wang, and L. Mandel. "Observation of nonlocal interference in separated photon channels". In: Phys. Rev. Lett. 65.3 (July 1990), pp. 321-324. DOI: 10.1103/physrevlett.65.321.

[28] P. Tapster, J. Rarity, and P. Owens. "Violation of Bell's Inequality over 4 km of Optical Fiber". In: Phys. Rev. Lett. 73.14 (Oct. 1994), pp. 1923-1926. DOI: 10.1103/physrevlett.73.1923.

[29] I. Marcikic, H. de Riedmatten, W. Tittel, H. Zbinden, M. Legré, and N. Gisin. "Distribution of Time-Bin Entangled Qubits over $50 \mathrm{~km}$ of Optical Fiber". In: Phys. Rev. Lett. 93.18 (Oct. 2004). DOI: 10.1103/ physrevlett.93.180502.

[30] T. Inagaki, N. Matsuda, O. Tadanaga, M. Asobe, and H. Takesue. "Entanglement distribution over $300 \mathrm{~km}$ of fiber". In: Opt. Express 21.20 (2013), p. 23241. DOI: 10.1364/oe. 21.023241.

[31] D. Grassani, S. Azzini, M. Liscidini, M. Galli, M. J. Strain, M. Sorel, J. E. Sipe, and D. Bajoni. "Micrometer-scale integrated silicon source of time-energy entangled photons". In: Optica 2.2 (2015), p. 88. DOI: 10.1364/optica.2.000088.

[32] P. A. M. Dirac. "A new notation for quantum mechanics". In: Mathematical Proceedings of the Cambridge Philosophical Society 35.03 (July 1939), p. 416. DOI: 10.1017/s0305004100021162. 
[33] M. Nielsen and I. Chuang. Quantum Computation and Quantum Information. Cambridge Series on Information and the Natural Sciences. Cambridge University Press, 2000. ISBN: 9780521635035.

[34] G. Brassard. "Brief history of quantum cryptography: A personal perspective". In: Theory and Practice in Information-Theoretic Security, 2005. IEEE Information Theory Workshop on. IEEE. 2005, pp. 19-23.

[35] J. S. Bell. "On the Einstein-Podolsky-Rosen paradox". In: Physics (Long Island City, N. Y.) 1 (1964), pp. 195-200.

[36] H. P. Stapp. "Bell's theorem and world process". In: Nuovo Cim B 29.2 (Oct. 1975), pp. 270-276. DOI: 10.1007/bf 02728310.

[37] A. Einstein, B. Podolsky, and N. Rosen. "Can Quantum-Mechanical Description of Physical Reality Be Considered Complete?" In: Physical Review 47.10 (May 1935), pp. 777-780. DOI: 10.1103/physrev . 47.777 .

[38] D. Bohm. Quantum Theory. Dover Books on Physics Series. PrenticeHall, 1951. ISBN: 9780486659695.

[39] W. Heisenberg. "Über den anschaulichen Inhalt der quantentheoretischen Kinematik und Mechanik". de. In: Zeitschrift für Physik 43.3-4 (Mar. 1927), pp. 172-198. ISSN: 0044-3328. DOI: 10.1007/ BF01397280.

[40] A. Einstein. "Quanten-Mechanik und Wirklichkeit". In: Dialectica 2.3-4 (Nov. 1948), pp. 320-324. DOI: $10.1111 /$ j . 1746-8361 . 1948. tb00704.x.

[41] J. Baggott. The Meaning of Quantum Theory: A Guide for Students of Chemistry and Physics (Oxford Science Publications). Oxford University Press, 1992. ISBN: 019855575X.

[42] N. Bohr. The Philosophical Writings of Niels Bohr. The Philosophical Writings of Niels Bohr. Ox Bow Press, 1934.

[43] J. F. Clauser, M. A. Horne, A. Shimony, and R. A. Holt. "Proposed Experiment to Test Local Hidden-Variable Theories". In: Phys. Rev. Lett. 23.15 (1969), pp. 880-884. DOI: 10.1103/PhysRevLett. 23. 880.

[44] P. Pearle. "Hidden-variable example based upon data rejection". In: Phys. Rev. D 2 (1970), pp. 1418-1425. DOI: 10.1103/PhysRevD. 2. 1418. 
[45] S. Braunstein and C. Caves. "Wringing out better Bell inequalities". In: Annals of Physics 202.1 (1990), pp. 22-56. DOI: 10.1016/00034916(90)90339-P.

[46] S. Pironio, A. Acín, N. Brunner, N. Gisin, S. Massar, and V. Scarani. "Device-independent quantum key distribution secure against collective attacks". In: New J. Phys. 11.4 (Apr. 2009), p. 045021. DOI: 10.1088/1367-2630/11/4/045021.

[47] P. Shor and J. Preskill. "Simple Proof of Security of the BB84 Quantum Key Distribution Protocol”. In: Phys. Rev. Lett. 85.2 (July 2000), pp. 441-444. DOI: 10.1103/physrevlett. 85. 441.

[48] D. Mayers. "Unconditional Security in Quantum Cryptography". In: J. ACM 48.3 (May 2001), pp. 351-406. ISSN: 0004-5411. DOI: 10. $1145 / 382780.382781$.

[49] V. Scarani. "The device-independent outlook on quantum physics". In: Acta Physica Slovaca 62.4 (2009), pp. 347-409. DOI: 10.2478/ v10155-012-0003-4.

[50] D. Mayers and A. Yao. "Quantum Cryptography with Imperfect Apparatus". In: Proceedings of the 39th Annual Symposium on Foundations of Computer Science. FOCS '98. Washington, DC, USA: IEEE Computer Society, 1998, pp. 503-. ISBN: 0-8186-9172-7.

[51] J. Barrett, L. Hardy, and A. Kent. "No Signaling and Quantum Key Distribution". In: Phys. Rev. Lett. 95 (2005), p. 010503. DOI: 10 . 1103/PhysRevLett.95.010503.

[52] A. Acín, N. Brunner, N. Gisin, S. Massar, S. Pironio, and V. Scarani. "Device-Independent Security of Quantum Cryptography against Collective Attacks". In: Phys. Rev. Lett. 98 (2007), p. 230501. DOI: 10.1103/PhysRevLett.98.230501.

[53] A. Acín, N. Gisin, and L. Masanes. "From Bell's Theorem to Secure Quantum Key Distribution”. In: Physical Review Letters 97.12 (Sept. 2006), p. 120405. DOI: 10.1103/PhysRevLett.97.120405.

[54] S. Popescu and D. Rohrlich. "Which states violate Bell's inequality maximally?" In: Physics Letters A 169.6 (Oct. 1992), pp. 411-414. DOI: $10.1016 / 0375-9601$ (92) 90819-8. 
[55] A. Cabello, J.-Å. Larsson, and D. Rodríguez. "Minimum detection efficiency required for a loophole-free violation of the BraunsteinCaves chained Bell inequalities". In: Phys. Rev. A 79 (6 June 2009), p. 062109. DOI: 10.1103/PhysRevA .79.062109.

[56] A. Aspect, P. Grangier, and G. Roger. "Experimental Tests of Realistic Local Theories via Bell's Theorem". In: Phys. Rev. Lett. 47.7 (Aug. 1981), pp. 460-463. DOI: 10.1103/physrevlett. 47.460.

[57] A. Aspect, J. Dalibard, and G. Roger. "Experimental Test of Bell's Inequalities Using Time-Varying Analyzers". In: Physical Review Letters 49.25 (Dec. 1982), pp. 1804-1807. DOI: 10.1103/PhysRevLett. 49.1804.

[58] J.- $\AA$. Larsson. "Loopholes in Bell inequality tests of local realism". In: Journal of Physics A 47.42 (Oct. 2014), p. 424003. ISSN: 1751-8121. DOI: $10.1088 / 1751-8113 / 47 / 42 / 424003$.

[59] A. Shimony, M. A. Horne, and J. F. Clauser. "Comment on "The theory of local beables"'. In: Epistemological letters 13.1 (1976).

[60] A. J. Leggett. In: Foundations of Physics 33.10 (2003), pp. 1469-1493. DOI: $10.1023 / \mathrm{a}: 1026096313729$.

[61] J. Bogdanski, J. Ahrens, and M. Bourennane. "Single mode fiber birefringence compensation in Sagnac and "plug \& play" interferometric setups". In: Opt. Express 17.6 (2009), p. 4485. DOI: 10 . 1364/ oe . 17.004485 .

[62] K. Borzycki and M. Jaworski. "Temperature Dependence of PMD in Optical Fibres and Cables ¿ Part II". In: 2006 International Conference on Transparent Optical Networks. IEEE, June 2006. DOI: 10.1109/icton. 2006.248541.

[63] A. M. Smith. "Polarization and magnetooptic properties of singlemode optical fiber". In: Applied Optics 17.1 (Jan. 1978), pp. 52-56. DOI: $10.1364 / \mathrm{AO} .17 .000052$.

[64] E. Frins and W. Dultz. "Rotation of the polarization plane in optical fibers". In: J. Lightwave Technol. 15.1 (1997), pp. 144-147. DOI: $10.1109 / 50.552122$.

[65] V. Hnizdo. "On Bohr's response to the clock-in-the-box thought experiment of Einstein". In: European journal of physics 23.4 (2002), pp. L9-L13. 
[66] J. Jogenfors and J.-Å. Larsson. "Energy-time entanglement, elements of reality, and local realism". In: Journal of Physics A 47.42 (Oct. 2014), p. 424032. ISSN: 1751-8121. DOI: $10.1088 / 1751-8113 / 47 /$ $42 / 424032$.

[67] A. Cabello, A. Rossi, G. Vallone, F. De Martini, and P. Mataloni. "Proposed Bell Experiment with Genuine Energy-Time Entanglement". In: Physical Review Letters 102 (Jan. 2009), p. 040401. DOI: 10.1103/PhysRevLett. 102.040401.

[68] G. Lima, G. Vallone, A. Chiuri, A. Cabello, and P. Mataloni. "Experimental Bell-inequality violation without the postselection loophole". In: Physical Review A 81.4 (Apr. 2010), p. 040101. DOI: 10.1103/ PhysRevA. 81.040101.

[69] J. Jogenfors, A. M. Elhassan, J. Ahrens, M. Bourennane, and J.- $\AA$. Larsson. "Hacking energy-time entanglement-based systems with classical light". Submitted and in review. Available as arXiv preprint arXiv:1411.7222. 2014.

[70] F. Träger. Springer Handbook of Lasers and Optics. Springer handbooks. Springer, 2007. ISBN: 9780387304205.

[71] V. Scarani and C. Kurtsiefer. "The black paper of quantum cryptography: Real implementation problems". In: Theoretical Computer Science 560 (Dec. 2014), pp. 27-32. DOI: 10 .1016/j . tcs . 2014. 09.015.

[72] S. Aerts, P. Kwiat, J.-Å. Larsson, and M. Zukowski. "Two-photon Franson-type experiments and local realism”. In: Physical Review Letters 83 (1999), pp. 2872-2875. ISSN: 00319007. DOI: 10.1103/ PhysRevLett.83.2872.

[73] M. Mafu, A. Marais, and F. Petruccione. "A Necessary Condition for the Security of Coherent-One-Way Quantum Key Distribution Protocol". In: Applied Mathematics \& Information Sciences 8.6 (Nov. 2014), pp. 2769-2773. DOI: 10.12785/amis/080612. 


\section{Glossary}

\section{Glossary}

Analysis station A device for manipulating and measuring quantum states. In our QKD, experiments, Alice and Bob have an analysis station each. $18,26,29,33,35,38,41-44,46$

BB84 The original QKD protocol by Bennett and Brassard from 1984. 7, 8, $18,21,31-35,39,41,48$

E91 Arthur Ekert's entanglement-based QKD protocol from 1991. 8, 18, $21,31-33,41,42$

Interferometer A system for testing quantum interference. In our QKD experiments, an interferometer represents the entire device with both Alice and Bob. 18, 28, 35, 44, 48

Schneier's Law Anyone, from the most clueless amateur to the best cryptographer, can create an algorithm that he himself can't break. 4, 47

\section{Acronyms}

CHSH Clauser, Horne, Shimony, Holt. 29, 30, 37, 42-44, 48

COW Coherent One-Way. 8, 48

DH Diffie-Hellman. 5, 6

DI-QKD Device-Independent Quantum Key Distribution. 33-36, 47 
DPS Distributed Phase-Shift. 8, 48

EPR Einstein, Podolsky, and Rosen. 23-26, 28, 30, 31

GCHQ Government Communications Headquarters. 5, 6

LHV Local Hidden Variable. 43, 44, 47

OTP one-time pad. 7, 17, 20, 21

QKD quantum key distribution. 7-9, 11, 15, 17, 18, 21, 26, 28, 31, 33-35, $39,41,42,47,48$

RNG random number generator. 19 


\section{Index}

Analysis station, 18

Beam splitter, 44

Bell test, 48

Bell inequality, 21, 27, 35, 36

Bell state, 23, 31, 35

Bell test, 33, 36

Bitcoin, 3

Black box model, 29, 33

Bra, 11

Bra-ket notation, 11

Check bits, 20, 21, 33, 42

Cipher, 3

Ciphertext, 3

Code, 3

Cryptanalysis, 2, 11, 47

Denial of service, 21

Detection efficiency, 37

Detection loophole, 36, 38, 43

Discrete logarithm, 6

Energy-time entanglement, 8, 39

Entanglement, 8, 31

Entanglement-based protocol, 8, 18, Path realism, 44 $21,33,34,39$

Factorization problem, 6

Faked Bell value, 43

Franson interferometer, 8, 18, 30, 40, 42-44, 46-48
Genuine energy-time entanglement, 44

Goppa code, 7

Hermitian conjugate, 11

Hidden variables, 25, 26, 29, 30, 36, 42

Information reconciliation, 21, 33, 42

Inner product, 15

Inner product, 11,12

Kerckhoff's principle, 4

Ket, 11

Key, 3

Locality, 25, 28, 29

Locality loophole, 38

Loophole, 35, 36, 38

Message, 3

One-way function, 5

Orthogonal vectors, 12

Pauli matrix, 19, 28

$\pi$ meson, 23, 24, 32

Plaintext, 3

Post-quantum cryptography, 7

Postselection, 41, 43

Postselection loophole, 43 
Prepare-and-measure protocol, 8,18 , 21,33

Privacy amplification, 21, 33, 42

Public-key cryptography, 5

Pure state, 12

Quantum algorithm, 6

Quantum channel, 7

Quantum computer, 6, 31, 47

Quantum teleportation, 31

Qubit, 6, 19, 27

Raw key, 20

Realism, 28, 29

RSA, 6

Schneier's Law, 4, 47

Security test, $21,33,35$

Security through obscurity, 5

Separable state, 31

Shor's algorithm, 6

Sifted key, 20

Skytale, 1

Source, 18, 31

State space, 12

State vector, 12, 15

Steganography, 3

Symmetric cryptography, 5

Tensor product, 13, 31

Trapdoor function, 5

Unconditional security, 7

Unitary map, 12-14 


\section{Publications}





\section{Publications}

The articles associated with this thesis have been removed for copyright reasons. For more details about these see:

http://urn.kb.se/resolve?urn=urn:nbn:se:liu:diva-114073 



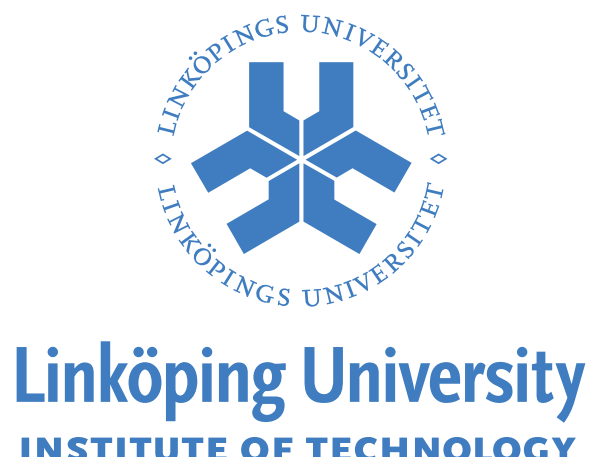

INSTITUTE OF TECHNOLOGY

Linköpings Universitet, 58183 Linköping, Sweden 